Article

\title{
Fatty Acid Biosynthesis in Chromerids
}

\author{
Aleš Tomčala ${ }^{1,2,+}{ }^{\oplus}$, Jan Michálek ${ }^{1,3,+}$, Ivana Schneedorferová ${ }^{1,3}$, Zoltán Füssy $^{1} \oplus$, \\ Ansgar Gruber ${ }^{1}$ (D), Marie Vancová ${ }^{1}$ and Miroslav Oborník ${ }^{1,3, * \mathbb{D}}$ \\ 1 Biology Centre CAS, Institute of Parasitology, Branišovská 31, 37005 České Budějovice, Czech Republic; \\ a.tomcala@centrum.cz (A.T.); jan.michalek@entu.cas.cz (J.M.); Ivana.Schneedorferova@email.cz (I.S.); \\ zoltan.fussy@gmail.com (Z.F.); ansgar.gruber@paru.cas.cz (A.G.); vancova@paru.cas.cz (M.V.) \\ 2 Faculty of Fisheries and Protection of Waters, CENAKVA, Institute of Aquaculture and Protection of Waters, \\ University of South Bohemia, Husova 458/102, 37005 České Budějovice, Czech Republic \\ 3 Faculty of Science, University of South Bohemia, Branišovská 31, 37005 České Budějovice, Czech Republic \\ * Correspondence: obornik@paru.cas.cz; Tel.: +420-38777-5464 \\ + These authors equally contributed to this work.
}

Received: 14 May 2020; Accepted: 15 July 2020; Published: 24 July 2020

check for updates

\begin{abstract}
Fatty acids are essential components of biological membranes, important for the maintenance of cellular structures, especially in organisms with complex life cycles like protozoan parasites. Apicomplexans are obligate parasites responsible for various deadly diseases of humans and livestock. We analyzed the fatty acids produced by the closest phototrophic relatives of parasitic apicomplexans, the chromerids Chromera velia and Vitrella brassicaformis, and investigated the genes coding for enzymes involved in fatty acids biosynthesis in chromerids, in comparison to their parasitic relatives. Based on evidence from genomic and metabolomic data, we propose a model of fatty acid synthesis in chromerids: the plastid-localized FAS-II pathway is responsible for the de novo synthesis of fatty acids reaching the maximum length of 18 carbon units. Short saturated fatty acids (C14:0-C18:0) originate from the plastid are then elongated and desaturated in the cytosol and the endoplasmic reticulum. We identified giant FAS I-like multi-modular enzymes in both chromerids, which seem to be involved in polyketide synthesis and fatty acid elongation. This full-scale description of the biosynthesis of fatty acids and their derivatives provides important insights into the reductive evolutionary transition of a phototropic algal ancestor to obligate parasites.
\end{abstract}

Keywords: Chromera velia; Vitrella brassicaformis; fatty acids; de novo biosynthesis; evolution; elongation; desaturation

\section{Introduction}

The phototrophic alveolates Chromera velia [1] and Vitrella brassicaformis [2] (referred to as chromerids) were isolated from stony corals in Australia using methods for the isolation of intracellular symbionts. Both chromerids can live as free-living algae without hosts in laboratory cultures; however, they are associated with corals in the wild [1-4]. Although they were found in the larvae of several coral species [5], they have never been detected in adult corals [6]. It was speculated that chromerids live as mutualists like symbiotic dinoflagellates of the genus Symbiodinium [1,7]. However, the latest transcriptomic survey of coral larvae experimentally infected by C. velia opens the possibility that the alga can be their facultative or even accidental parasite [8]. The environmental detection of uncultured and undescribed chromerids and related organisms (ARL-V) also implies their probable commensal or parasitic relationship to corals [9]. Such finding is congruent with the accumulating evidence supporting the common ancestry of phototrophic chromerids and parasitic apicomplexans [3,4,10-14]. Analyses of plastid [11] and mitochondrial [15] and nuclear genomes [10,13], together with the determination of ultrastructural characters such as cortical alveoli, four-membrane 
plastid envelopes [1], the presence of a pseudoconoid in C. velia [16], and zoosporogenesis by budding in $V$. brassicaformis [17], support the unique phylogenetic position of chromerids at the root of the parasitic Apicomplexa. Further molecular phylogenetic and phylogenomic analyses have placed the predatory colpodellids within the polyphyletic chromerids (Figure 1), constituting a group named Apicomonada [18] or chrompodellids [10].

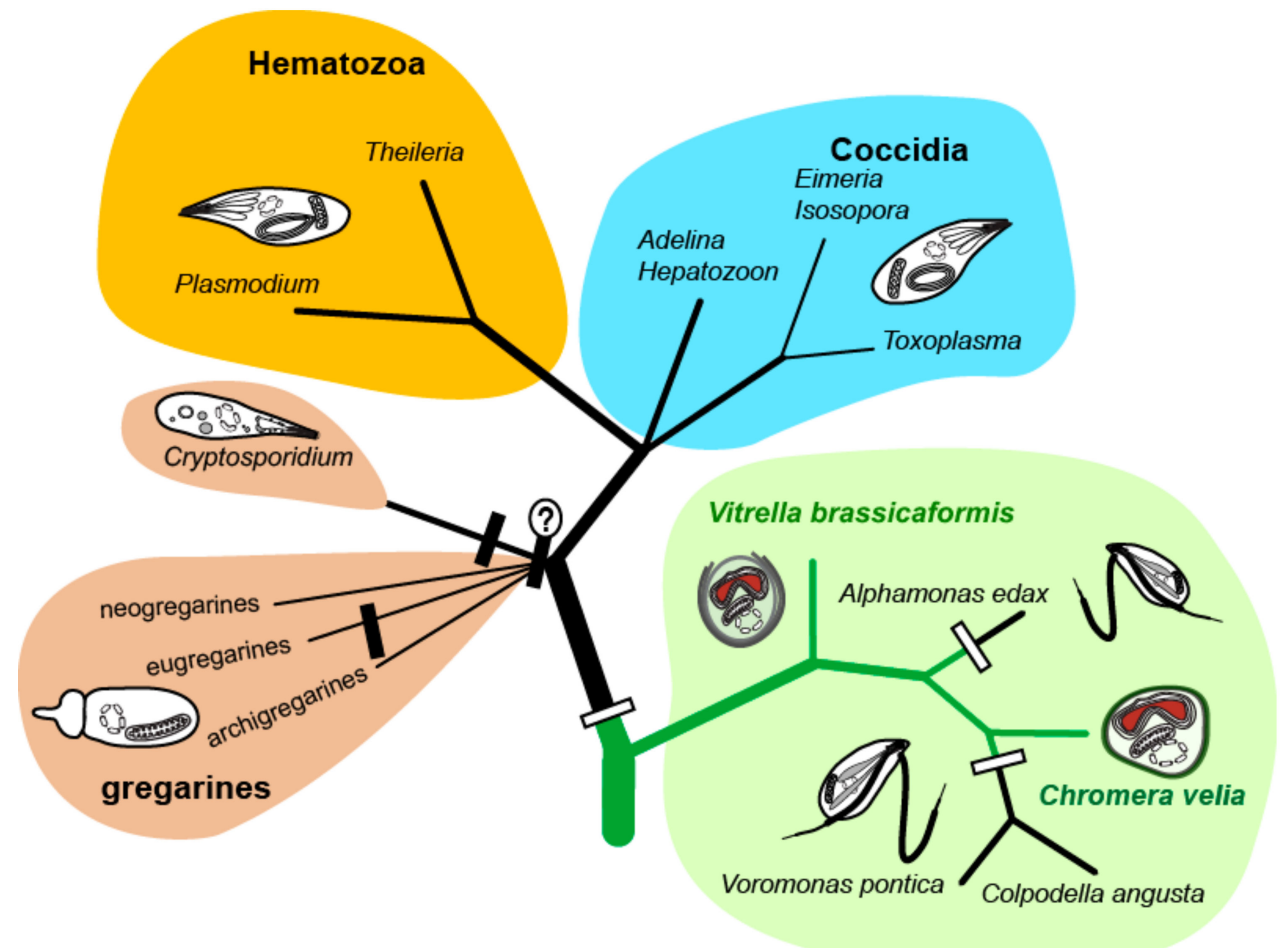

Figure 1. Phylogenetic tree of chromerid algae and apicomplexan parasites. The white rectangles indicate the loss of photosynthesis, the black ones indicate the loss of plastid.

Fatty acids (FAs), carboxylic acids with aliphatic chains, represent a fundamental component of biological lipids and can be divided into several categories according to their structure [19]. Here, we focus on the most abundant subclass-straight-chained saturated and unsaturated FAs with a terminal carboxylic acid group. Generally, FAs are synthesized by repeated addition of two-carbon units to a growing carboxylic acid chain attached to the acyl carrier protein (ACP) [20]. In cellular organisms, the enzymes responsible for the de novo synthesis of FAs are either the type I FA synthases (FAS-I) typically found in heterotrophic eukaryotes or the type II FA synthases (FAS-II) usually referred to as bacterial-type FAS-II. Both pathways contain identical reaction steps but they substantially differ in the architecture of the responsible enzymes. In eukaryotes, cytosolic FAS-I is a huge multi-modular enzyme [21], while FAS-II consists of individual separate enzymes located in the plastid [22-24]. Modules in the FAS-I multi-modular enzymes have the full set of domains needed for the attachment of FA carbon units. If such a multi-modular enzyme contains an incomplete module lacking some of the domains (often acyltransferase) or has some additional domains (e.g., methyltransferase), it usually has a different function. Such giant multi-modular enzymes known as polyketide synthases (PKSs) are responsible for the synthesis of antimicrobial compounds called polyketides (PK) [25]. It was proposed that FAS-I derived from FAS-II via a gene fusion [25]. Plants and algae primarily use bacterial FAS-II located in the plastid, with the involved enzymes encoded in the nuclear genome [24,26]. Many algae also possess FAS-I/PKS-like enzymatic equipment. Their function is still enigmatic; however, they likely do not act as FA synthases [25]. Various lineages of apicomplexan parasites differ in the presence of the particular FAS system. Most apicomplexan parasites possess the apicoplast-located FAS-II for de novo production of FAs [20,27,28], with Haemosporidia such as 
Plasmodium utilizing only this prokaryotic type of the synthesis. The coccidians Toxoplasma gondii or Eimeria tenella were described to use the apicoplast FAS-II system and the cytosolic FAS-I in parallel, with life stage-specific FA metabolism [20,29-31]. However, the apicomplexan minimalists Theileria parva and Babesia bigemina completely lost all the enzymes for FA synthesis and scavenge all FAs from their hosts [20]. Current findings suggest independent multiple FAS-II losses in apicomplexans and their relatives [32].

Last but not least, intestinal parasites of the genus Cryptosporidium do not possess the apicoplast at all and lack any de novo FA synthesis. The huge FAS-I-like multi-modular enzyme coded in the Cryptosporidium parvum genome is responsible for the elongation of palmitic acid acquired from the host $[13,20,33]$. The high divergence of the apicomplexan sources of fatty acids reflects different stages of reductive evolution driven by adaptations to diverse hosts [13]. Genomic analysis performed by John and co-workers [34] revealed that some representatives among apicomplexans such as C. parvum, E. tenella, and T. gondii possess PKS genes. However, the presence of PKS genes is not universal among apicomplexans. For instance, in the genomes of Plasmodium falciparum and T. parva, these sequences are lacking [34].

Here, we studied FAs and their synthesis in the chromerids C. velia and V. brassicaformis by the combination of analytical chemistry and genomics methods. We compared chromerid and apicomplexan FA biosynthesis and propose a scenario of their evolution.

\section{Materials and Methods}

\subsection{Growth Experiments}

Cultures of $C$. velia and $V$. brassicaformis were grown in $\mathrm{f} / 2$ medium in $25 \mathrm{~cm}^{2}$ flasks under artificial light, with a photoperiod of $12 \mathrm{~h}$ light/12 h dark and light intensities of $30-50 \mu \mathrm{mol} \mathrm{m}^{-2} \mathrm{~s}^{-1}$ at a temperature of $26^{\circ} \mathrm{C}$. As an inoculum, $1 \mathrm{~mL}$ of $C$. velia $\left(1 \times 10^{6}\right.$ cells $\left./ \mathrm{mL}\right)$ or $5 \mathrm{~mL}$ of $V$. brassicaformis $\left(0.3 \times 10^{6}\right.$ cells $\left./ \mathrm{mL}\right)$ stationary culture was added to each flask with $40 \mathrm{~mL}$ of $\mathrm{f} / 2 \mathrm{medium}$. The cultures were grown for 50 days and harvested via centrifugation. Pellets for lipid extraction were stored at $-20^{\circ} \mathrm{C}$.

The cultures of C. velia were treated with Triclosan (TCI Europe N. V., Zwijndrecht, Belgium) [35] in the same cultivations conditions as mentioned above. Triclosan was diluted in dimethyl sulfoxide (DMSO, Sigma Aldrich, St. Louis, Missouri, USA). The inhibitor was added to cultures after 14 days, at six concentrations in the media $(0.02,0.04,0.08,0.133,0.333$, and $0.667 \mu \mathrm{M})$. The densities of the cultures were periodically measured by a spectrophotometer (Infinite ${ }^{\circledR} 200$ PRO, Tecan, Männedorf, Switzerland) over the course of 36 days. Fifty-day-old cultures were harvested by centrifugation in $50 \mathrm{~mL}$ flacons (Hettich Micro 22R centrifuge with rotor radium $100 \mathrm{~mm}$, set to $6000 \mathrm{rpm}$ at the of temperature $4{ }^{\circ} \mathrm{C}$ ). The obtained pellets were split; the first part was stored at $-20{ }^{\circ} \mathrm{C}$ for fatty acid analyses; the second part was subjected to transmission electron microscopy (TEM).

To investigate the effects of various levels of inorganic nitrogen in the media, C. velia cultures were grown in standard conditions, as mentioned above (light, temperature, cultivation flasks). Flasks containing $120 \mathrm{~mL}$ of $\mathrm{f} / 2$ solution with four different concentrations of $\mathrm{NaNO}_{3}$ (from 0.000 to 0.062 $\mathrm{g}$ of pure nitrogen per $1 \mathrm{~L}$ of $\mathrm{f} / 2$ medium) were inoculated using $1 \mathrm{~mL}$ of stationary-stage culture of C. velia $\left(1 \times 10^{6}\right.$ cells $\left./ \mathrm{mL}\right)$. The highest concentration of nitrate in this experiment was five times higher than in the regular $\mathrm{f} / 2$ medium. The cultures were harvested by centrifugation after 30 days. The dry pellets were weighed and investigated using gas chromatography coupled with a flame ionization detector (GC/FID).

\subsection{Genomic Search}

BLAST search and InterProScan in the publicly available dataset of predicted proteins [13] (CryptoDB; https://cryptodb.org/cryptodb/) were used to search for the enzymes of the FA biosynthesis in C. velia and V. brassicaformis. The obtained sequences were complemented with homologs found in 
other public databases (NCBI, KEGG, JGI) and aligned using MAFFT [36]. The alignment was manually edited by excluding gaps and ambiguously aligned positions using Geneious [37]. Phylogenetic trees were computed using the maximum likelihood method (RAxML; [38] and Bayesian inference (MrBayes 3.2.4.; [39,40]. The subcellular locations of the enzymes were predicted using TargetP [41,42], SignalP [43], and ASAFind [44]. Localizations were established with the support of multiple predictors together; previously published data were also considered. For phylogenetic analyses, we took advantage of computational resources freely provided by the CIPRES Science Gateway [45].

\subsection{Fatty Acids_Gas Chromatography Coupled with a Flame Ionization Detector (GC/FID)}

Lipids were extracted following the chloroform-methanol solution method [46] modified by Košt ál and Šimek [47], with additions described by Tomčala et al. [48]. Briefly, homogenized algal samples were dried, weighed, and extracted. The raw extracts in chloroform were dried and resolved in $500 \mu \mathrm{L}$ of chloroform. Then, $100 \mu \mathrm{L}$ aliquots were transformed into methyl esters of fatty acids (FAMEs) to allow for GC analyses. For this purpose, sodium methoxide was employed as a transesterification reagent, as described previously [49]. FAMEs were then analyzed by GC/FID using a gas chromatograph GC-2014 (Shimadzu, Shimadzu Scientific Instruments Inc., Kyoto, Japan) equipped with the column BPX70 (SGE, SciTech, Prague, Czech Republic) and an autosampler with the injector AOC-20i (Shimadzu), with the settings according to a previous work published by Tomčala et al. [48]. For the identification of FAs, a mixture of 37 standards purchased from Supelco Inc. (Bellefonte, Pennsylvania, USA) was used.

\subsection{Transmission Electron Microscopy}

Pellets were transferred to HPF (High Pressure Freeze) carriers (Leica Microsyst. Vienna, Austria), then $\mathrm{f} / 2$ medium with $20 \%(w / v)$ bovine serum albumin (BSA) was added to fill the carriers completely. The samples were immediately frozen using the high-pressure freezer Leica EM PACT2. The frozen samples were incubated with $2 \%$ osmium diluted in $100 \%$ acetone in a freeze substitution unit, with the following substitution program: $-90^{\circ} \mathrm{C}$ for $96 \mathrm{~h}$; then the temperature was increased to $-20^{\circ} \mathrm{C}$ (with a slope of $\left.5{ }^{\circ} \mathrm{C} / \mathrm{h}\right)$; after $24 \mathrm{~h}$, the temperature was raised $\left(3^{\circ} \mathrm{C} / \mathrm{h}\right)$ to the final temperature of $4{ }^{\circ} \mathrm{C}$. After $18 \mathrm{~h}$ of incubation, the samples were washed with acetone and infiltrated by $25 \%, 50 \%, 75 \%$ EMBed 812 resin (EMS)/acetone solutions, $1 \mathrm{~h}$ at each step, at room temperature. Finally, fresh resin was added, and the specimens were placed overnight in a vacuum desiccator. The resin was allowed to polymerize at $60^{\circ} \mathrm{C}$ for $48 \mathrm{~h}$. Images were obtained by a transmission electron microscope (JEM 1010, JEOL Inc., USA) with an acceleration voltage of $100 \mathrm{kV} \mathrm{[16].}$

\section{Results}

\subsection{De novo Fatty Acid Synthesis}

The presence of a FAS-II in C. velia was first described by Dahmen et al. [50] and Woo et al. [13], who indicated the presence of FAS-I and FAS-II in both chromerids. We searched in the chromerid genomic and transcriptomic data (CryptoDB) for all the enzymes possibly involved in the synthesis of FAs and their derivatives (Tables 1-5). In addition to the canonical FAS-I and FAS-II enzymes, the genome contains a large number of genes likely encoding ketoacyl synthases (36 genes), short-chain dehydrogenases (73), or acyltransferase domains (29), present in various gene arrangements, often in a form of multi-modular enzymes with unknown putative function in FA or PK synthesis (Tables 1-5). We performed a phylogenetic analysis based on ketoacyl synthase (KS) domains to distinguish FAS-I and FAS-II enzymes of chromerids and further calculated detailed phylogenies of FAS-I- and FAS-II-type enzymes (Figures 2-4). Both chromerids possess numerous giant multi-modular enzymes in sizes ranging from 1000 to 11,000 amino acids composed of 1-5 modules and classified as putative FAS-I/PKS-I [13]. In general, KSs of type II FAS show two main lineages, i.e., $\alpha$-proteobacterial and cyanobacterial, with the latter genes involved in plastid-located KSs in chromerids (Figure 3). 
Ketoacyl synthases from FAS-I/PKS-I enzymes of chromerids, apicomplexans, and dinoflagellates form a sister group to PKSs responsible for the production of polyketide-based algal toxins found in dinoflagellates, chlorophytes, haptophytes, and stramenopiles (Figure 4). Since the evolutionary conversion between FAS and PKS function can be caused by a single amino acid substitution affecting the functionality of the participating domain [51], it is hard to identify them just by a phylogenetic signal. One distant clade of type I multi-modular FAS-like enzymes is formed by two genes of $C$. velia (Cvel_8275.t1, Cvel_22311.t1). These two genes share a multiple-domain architecture reminding a single module of PKS as well but they are interestingly fused with an N-terminal structure related to the AcrB-like domain family (multidrug efflux pump) or the sterol-sensing domain, which, together with a predicted N-terminal signal anchor (Tables 1-5) implies a probable association of the enzyme with the endoplasmic reticulum (ER).

Table 1. Ketoacyl synthases (KSs), elongases, and desaturases in chromerids and their predicted subcellular location. ER-endoplasmic reticulum.

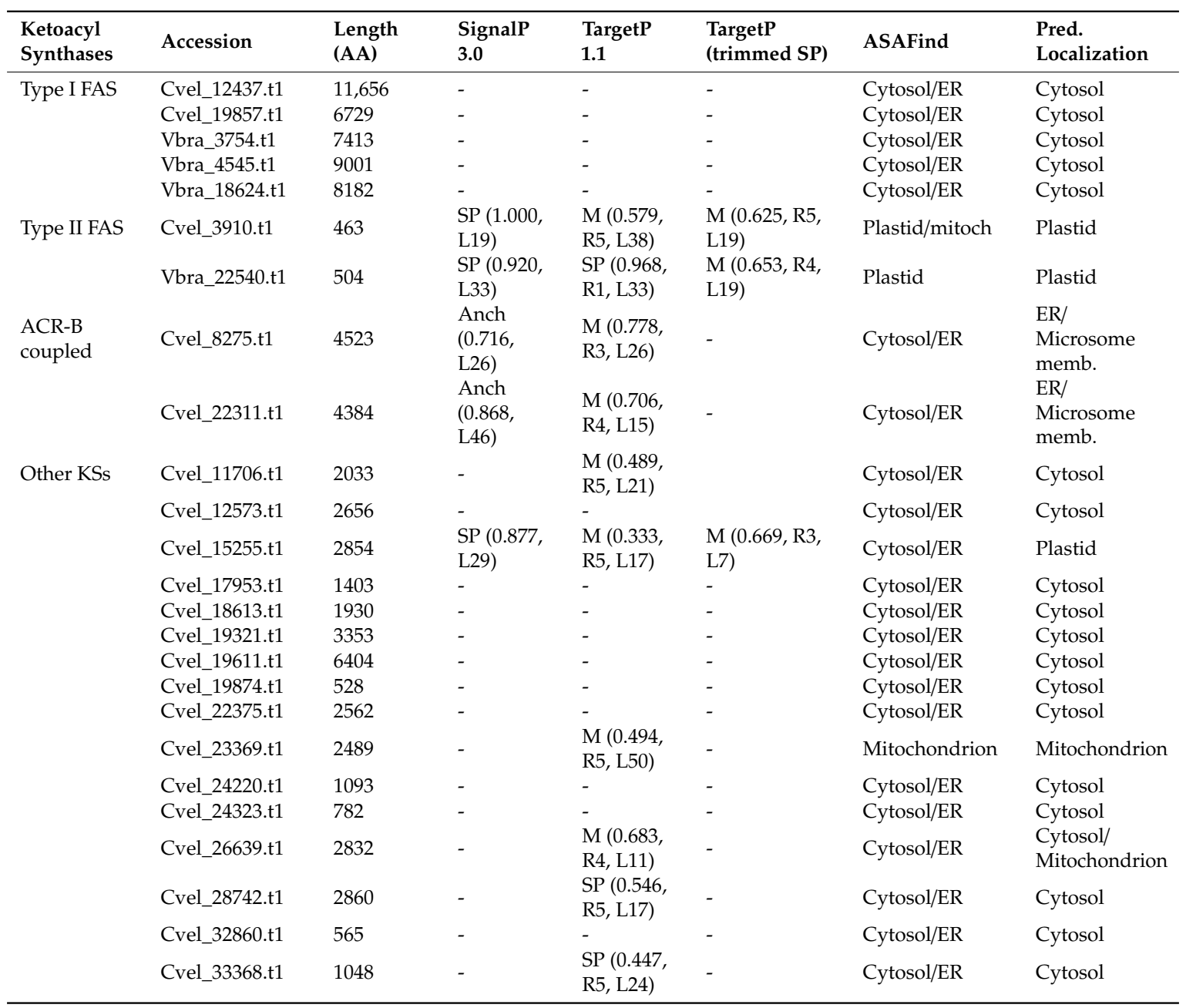


Table 2. Ketoacyl synthases, elongases, and desaturases in chromerids and their predicted subcellular location.

\begin{tabular}{|c|c|c|c|c|c|c|c|}
\hline Ketoacyl Synthases & Accession & Length (AA) & SignalP 3.0 & TargetP 1.1 & TargetP (trimmed SP) & ASAFind & Pred. Localization \\
\hline \multirow[t]{25}{*}{ Other KSs } & Cvel_33369.t1 & 720 & - & - & - & Cytosol/ER & Cytosol \\
\hline & Cvel_33747.t1 & 1598 & - & - & - & Cytosol/ER & Cytosol \\
\hline & Cvel_3424.t1 & 4498 & - & - & - & Cytosol/ER & Cytosol \\
\hline & Cvel_34436.t1 & 1211 & - & - & - & Cytosol/ER & Cytosol \\
\hline & Cvel_34654.t1 & 1165 & - & - & - & Cytosol/ER & Cytosol \\
\hline & Cvel_34826.t1 & 1174 & - & - & - & Cytosol/ER & Cytosol \\
\hline & Cvel_35138.t1 & 1013 & - & - & - & Cytosol/ER & Cytosol \\
\hline & Cvel_35394.t1 & 1007 & - & - & - & Cytosol/ER & Cytosol \\
\hline & Cvel_35394.t2 & 968 & - & - & - & Cytosol/ER & Cytosol \\
\hline & Cvel_36416.t1 & 675 & - & - & - & Cytosol/ER & Cytosol \\
\hline & Cvel_36618.t1 & 349 & - & - & - & Cytosol/ER & Cytosol \\
\hline & Cvel_3734.t1 & 4498 & - & - & - & Cytosol/ER & Cytosol \\
\hline & Cvel_6161.t1 & 6550 & - & - & - & Cytosol/ER & Cytosol \\
\hline & Cvel_6212.t1 & 2635 & - & - & - & Cytosol/ER & Cytosol \\
\hline & Cvel_678.t1 & 2469 & - & - & - & Cytosol/ER & Cytosol \\
\hline & Vbra_1266.t1 & 6691 & - & - & - & Cytosol/ER & Cytosol \\
\hline & Vbra_1193.t1 & 1857 & - & - & - & Cytosol/ER & Cytosol \\
\hline & Vbra_12045.t1 & 3268 & - & - & - & Cytosol/ER & Cytosol \\
\hline & Vbra_12047.t1 & 1204 & - & SP $(0.777, \mathrm{R} 4, \mathrm{~L} 19)$ & - & Cytosol/ER & Cytosol \\
\hline & Vbra_135.t1 & 1603 & - & - & - & Cytosol/ER & Cytosol \\
\hline & Vbra_14267.t1 & 1337 & - & - & - & Cytosol/ER & Cytosol \\
\hline & Vbra_1496.t1 & 3575 & - & - & - & Cytosol/ER & Cytosol \\
\hline & Vbra_16257.t1 & 1220 & - & - & - & Cytosol/ER & Cytosol \\
\hline & Vbra_21227.t1 & 6651 & - & - & - & Cytosol/ER & Cytosol \\
\hline & Vbra_2242.t1 & 995 & SP $(0.959$, L16) & $\mathrm{M}(0.442, \mathrm{R} 5, \mathrm{~L} 12)$ & - & Cytosol/ER & $\begin{array}{l}\text { ER/Microsome } \\
\text { lumen }\end{array}$ \\
\hline
\end{tabular}


Table 3. Ketoacyl synthases, elongases, and desaturases in chromerids and their predicted subcellular location.

\begin{tabular}{|c|c|c|c|c|c|c|c|}
\hline Ketoacyl Synthases & Accession & Length (AA) & SignalP 3.0 & TargetP 1.1 & TargetP (trimmed SP) & ASAFind & Pred. Localization \\
\hline \multirow[t]{19}{*}{ Other KSs } & Vbra_22449.t1 & 1668 & - & - & - & Cytosol/ER & Cytosol \\
\hline & Vbra_23040.t1 & 4167 & - & - & - & Cytosol/ER & Cytosol \\
\hline & Vbra_23145.t1 & 480 & - & - & - & Cytosol/ER & Cytosol \\
\hline & Vbra_23180.t1 & 2233 & - & - & - & Cytosol/ER & Cytosol \\
\hline & Vbra_23216.t1 & 301 & - & - & - & Cytosol/ER & Cytosol \\
\hline & Vbra_23250.t1 & 1732 & - & - & - & Cytosol/ER & Cytosol \\
\hline & Vbra_23378.t1 & 1092 & - & - & - & Cytosol/ER & Cytosol \\
\hline & Vbra_424.t1 & 1469 & - & - & - & Cytosol/ER & Cytosol \\
\hline & Vbra_425.t1 & 1098 & - & - & - & Cytosol/ER & Cytosol \\
\hline & Vbra_428.t1 & 3041 & - & - & - & Cytosol/ER & Cytosol \\
\hline & Vbra_460.t1 & 3015 & - & - & - & Cytosol/ER & Cytosol \\
\hline & Vbra_483.t1 & 2820 & - & $\mathrm{SP}(0.479, \mathrm{R} 4, \mathrm{~L} 43)$ & - & Cytosol/ER & Cytosol \\
\hline & Vbra_50.t1 & 526 & - & - & - & Cytosol/ER & Cytosol \\
\hline & Vbra_502.t1 & 710 & SP $(0.871, \mathrm{~L} 20)$ & $\mathrm{SP}(0.479, \mathrm{R} 4, \mathrm{~L} 20)$ & - & Plastid & Plastid/ER \\
\hline & Vbra_51.t1 & 3829 & - & - & - & Cytosol/ER & Cytosol \\
\hline & Vbra_6248.t1 & 1297 & - & - & - & Cytosol/ER & Cytosol \\
\hline & Vbra_7032.t1 & 2189 & - & - & - & Cytosol/ER & Cytosol \\
\hline & Vbra_7414.t1 & 461 & - & - & - & Cytosol/ER & Cytosol \\
\hline & Vbra_8741.t1 & 1101 & - & - & - & Cytosol/ER & Cytosol \\
\hline FASII (remaining) parts) & Accession & Length (AA) & SignalP 3.0 & TargetP 1.1 & TargetP (trimmed SP) & ASAFind & Pred. Localization \\
\hline \multirow{2}{*}{ Acyl transferase } & Cvel_4616.t1 & 349 & SP $(0.983$, L20) & SP $(0.729, \mathrm{~L} 19, \mathrm{R} 3)$ & M 0.526 (R5, L19) & Plastid & Plastid \\
\hline & Vbra_21812.t1 & 362 & $\mathrm{SP}(0.996, \mathrm{~L} 21)$ & $\mathrm{SP}(0.694, \mathrm{~L} 20, \mathrm{R} 3)$ & M 0.635 (R4, L15) & Plastid & Plastid \\
\hline \multirow[t]{2}{*}{ Enoyl reductase } & Cvel_5563.t1 & 355 & $\mathrm{SP}(0.958, \mathrm{~L} 20)$ & $\mathrm{SP}(0.785, \mathrm{~L} 19, \mathrm{R} 3)$ & M 0.912 (R2, L23) & Plastid & Plastid \\
\hline & Vbra_11747.t1 & 412 & SP $(0.996$, L22) & SP $(0.982, \mathrm{~L} 33, \mathrm{R} 1)$ & M 0.919 (R1, L20) & Cytosol/ER & Plastid \\
\hline \multirow[t]{2}{*}{ Ketoacyl reductase (KR) } & Cvel_3619.t1 & 392 & SP $(0.912$, L19) & $\mathrm{SP}(0.915, \mathrm{~L} 18, \mathrm{R} 1)$ & M 0.700 (R3, L17) & Plastid & Plastid \\
\hline & Vbra_710.t1 & 338 & SP (0.996, L22) & $\mathrm{SP}(0.943, \mathrm{~L} 21, \mathrm{R} 1)$ & M 0.766 (R3, L10) & Plastid & Plastid \\
\hline \multirow[t]{2}{*}{ Dehydrogenase } & Cvel_14912.t1 & 213 & SP $(0.849, \mathrm{~L} 18)$ & $\mathrm{SP}(0.884, \mathrm{~L} 17, \mathrm{R} 2)$ & M 0.526 (R5, L19) & Plastid & Plastid \\
\hline & Vbra_19455.t1 & 225 & SP (0.993, L25) & $\mathrm{SP}(0.800, \mathrm{~L} 22, \mathrm{R} 2)$ & M 0.827 (R2, L43) & Plastid & Plastid \\
\hline
\end{tabular}


Table 4. Ketoacyl synthases, elongases, and desaturases in chromerids and their predicted subcellular location. S/MUFAs, saturated and monounsaturated FAs, PUFA, poly-unsaturated FAs.

\begin{tabular}{|c|c|c|c|c|c|c|c|}
\hline \multicolumn{8}{|l|}{ Elongases } \\
\hline \multirow[t]{10}{*}{ S/MUFA } & Cvel_6334.t1 & 193 & $\mathrm{SP}(0.960, \mathrm{~L} 16)$ & $\mathrm{SP}(0.861, \mathrm{R} 2, \mathrm{~L} 16)$ & M (0.689, R3, L5) & Cytosol/ER & Plastid \\
\hline & Cvel_13090.t1 & 525 & Anch $(0.838, \mathrm{~L} 41)$ & - & - & Cytosol/ER & ER/Microsome memb. \\
\hline & Cvel_14249.t1 & 884 & Anch $(0.791$, L39) & - & - & Cytosol/ER & ER/Microsome memb. \\
\hline & Cvel_15641.t1 & 2131 & Anch $(0.606, \mathrm{~L} 50)$ & - & - & Cytosol/ER & ER/Microsome memb. \\
\hline & Cvel_12461.t1 & 415 & - & - & - & Cytosol/ER & Cytosol \\
\hline & Vbra_3929.t1 & 307 & - & - & - & Cytosol/ER & Cytosol \\
\hline & Vbra_9666.t1 & 885 & $\mathrm{SP}(0.675, \mathrm{~L} 24)$ & $\mathrm{SP}(0.622, \mathrm{R} 3, \mathrm{~L} 40)$ & $\mathrm{S}(0.950, \mathrm{R} 1, \mathrm{~L} 16)$ & Cytosol/ER & ER/Microsome lumen \\
\hline & Vbra_11085.t1 & 281 & Anch $(0.560, \mathrm{~L} 16)$ & - & - & Cytosol/ER & ER/Microsome memb. \\
\hline & Vbra_12050.t1 & 330 & Anch $(0.600, \mathrm{~L} 41)$ & - & - & Cytosol/ER & ER/Microsome memb. \\
\hline & Vbra_13163.t1 & 363 & - & - & - & Cytosol/ER & Cytosol \\
\hline \multirow[t]{4}{*}{ PUFA } & Cvel_1604.t1 & 297 & - & - & - & Cytosol/ER & Cytosol \\
\hline & Vbra_3441.t1 & 363 & Anch $(0.984$, L55) & - & - & Cytosol/ER & ER/Microsome memb. \\
\hline & Vbra_11843.t1 & 306 & Anch $(0.986, \mathrm{~L} 63)$ & - & - & Cytosol/ER & ER/Microsome memb. \\
\hline & Vbra_16961.t1 & 291 & Anch $(0.638, \mathrm{~L} 62)$ & - & - & Cytosol/ER & ER/Microsome memb. \\
\hline
\end{tabular}


Table 5. Ketoacyl synthases, elongases, and desaturases in chromerids and their predicted subcellular location.

\begin{tabular}{|c|c|c|c|c|c|c|c|}
\hline Desaturases & Accession & Length (AA) & SignalP 3.0 & TargetP 1.1 & TargetP (trimmed SP) & ASAFind & Pred. Localization \\
\hline \multirow[t]{6}{*}{ Omega } & Cvel_2615.t1 & 461 & - & - & - & Cytosol/ER & Cytosol \\
\hline & Cvel_21003.t1 & 440 & - & - & - & Cytosol/ER & Cytosol \\
\hline & Cvel_22707.t1 & 425 & SP $(0.999, \mathrm{~L} 17)$ & M/SP (0.631/0.771,L17, R5) & $\mathrm{M}(0.852, \mathrm{R} 2, \mathrm{~L} 71)$ & Plastid & Plastid \\
\hline & Vbra_7407.t1 & 445 & - & $\mathrm{M}(0.564, \mathrm{~L} 12, \mathrm{R} 5)$ & - & Cytosol/ER & Cytosol \\
\hline & Vbra_15192.t1 & 465 & - & - & - & Cytosol/ER & Cytosol \\
\hline & Vbra_20615.t1 & 447 & - & - & - & Cytosol/ER & Cytosol \\
\hline \multirow[t]{4}{*}{ Delta 9} & Cvel_14249.t1 & 884 & Anch $(0.791$, L39) & - & - & Cytosol/ER & ER/Microsome memb. \\
\hline & Cvel_21149.t1 & 395 & SP $(0.910$, L37) & $\mathrm{SP}(0.979, \mathrm{~L} 37, \mathrm{R} 1)$ & M 0.814 (R2, L6) & Cytosol/ER & Plastid \\
\hline & Vbra_9666.t1 & 885 & $\mathrm{SP}(0.675, \mathrm{~L} 40)$ & $\mathrm{SP}(0.622, \mathrm{~L} 40, \mathrm{R} 3)$ & - & Cytosol/ER & ER/Microsome lumen \\
\hline & Vbra_15445.t1 & 565 & - & M $(0.549$, L19, R5) & - & Cytosol/ER & Cytosol \\
\hline \multirow[t]{4}{*}{ Front-end } & Cvel_8966.t1 & 507 & - & - & - & Cytosol/ER & Cytosol \\
\hline & Cvel_17413.t1 & 543 & - & - & - & Cytosol/ER & Cytosol \\
\hline & Vbra_17659.t1 & 439 & - & - & - & Cytosol/ER & Cytosol \\
\hline & Vbra_20473.t1 & 437 & - & M $(0.738, \mathrm{~L} 17, \mathrm{R} 3)$ & - & Mitochondrion & Mitochondrion \\
\hline \multirow[t]{5}{*}{ Acetyl-CoA Carboxylases } & Accession & Length (AA) & SignalP 3.0 & TargetP 1.1 & TargetP (trimmed SP) & ASAFind & Pred. Localization \\
\hline & Cvel_530.t2 & 2097 & - & $\mathrm{M}(0.478, \mathrm{~L} 18, \mathrm{R} 5)$ & - & Cytosol/ER & Cytosol \\
\hline & Cvel_25292.t1 & 1651 & - & - & - & Cytosol/ER & Cytosol \\
\hline & Vbra_9562.t1 & 2702 & - & - & - & Cytosol/ER & Cytosol \\
\hline & Vbra_15163.t1 & 2146 & SP $(0.999$, L19) & $\mathrm{SP}(0.828, \mathrm{~L} 19, \mathrm{R} 2)$ & M 0.833 (R2, L20) & Cytosol/ER & Plastid \\
\hline
\end{tabular}




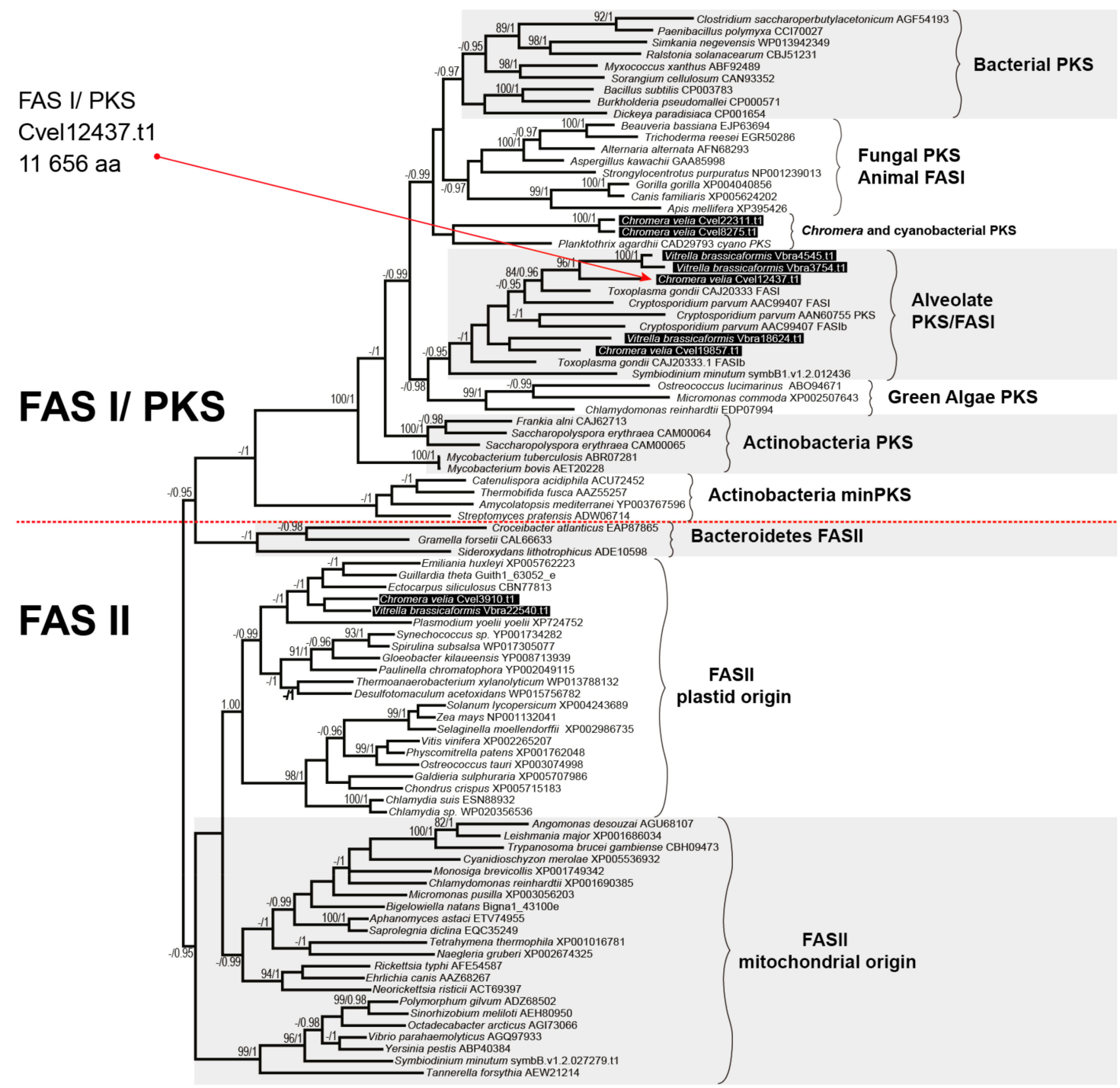

Figure 2. The phylogenetic tree as inferred from ketoacyl synthase domains to distinguish type I and type II fatty acid synthases. Bootstrap for 1000 repetitions and Bayesian pp values (BS1000/1M, CAT/LG models) are displayed above or below the branches. FAS, fatty acid synthase, PKS, polyketide synthase. 


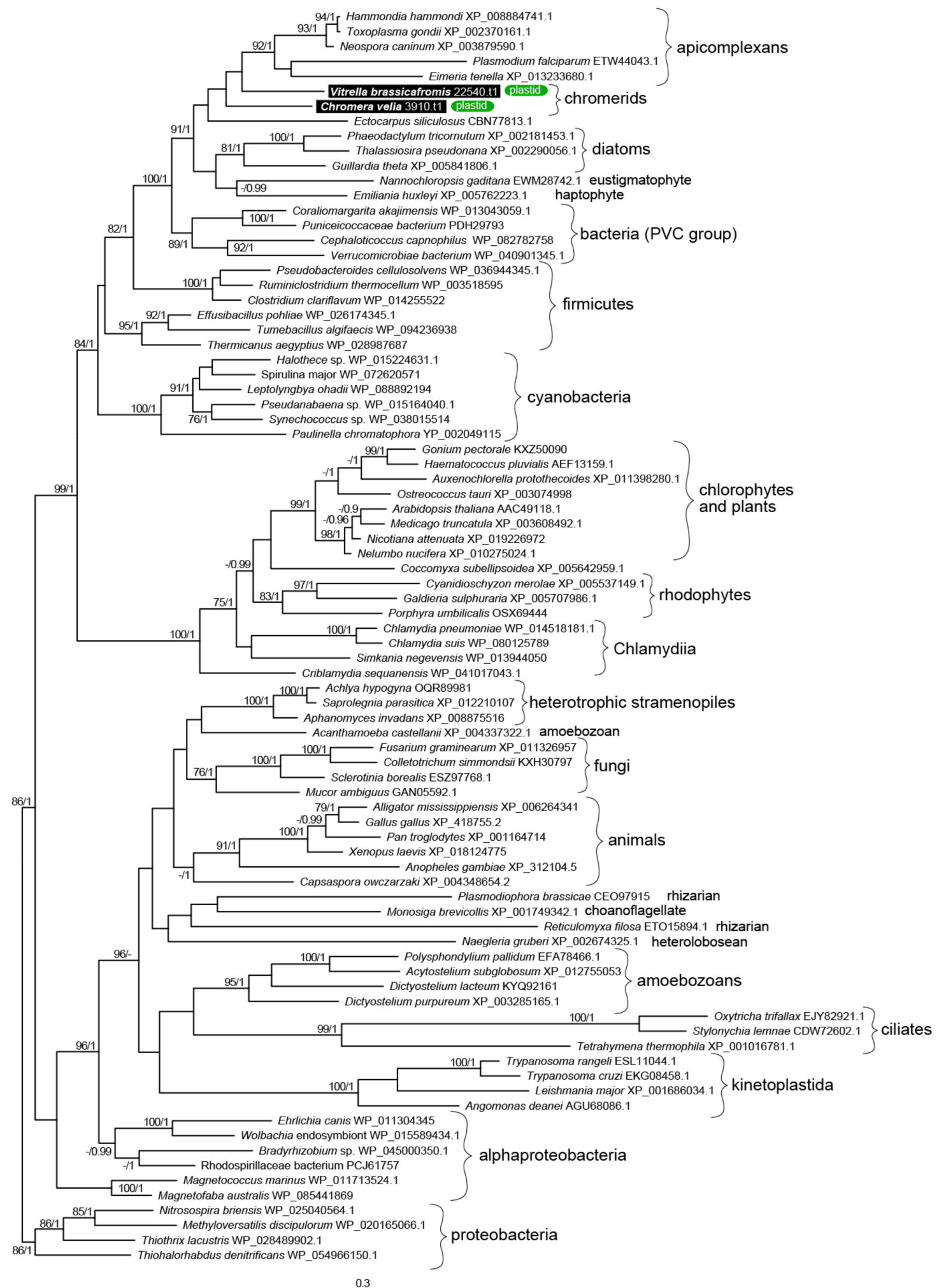

Figure 3. The detailed maximum likelihood phylogeny of ketoacyl synthase domains of type II fatty acid synthases. Bootstrap for 1000 repetitions and Bayesian pp values (BS1000/1M, CAT/LG models) are displayed above the branches. Predicted subcellular locations are shown. 


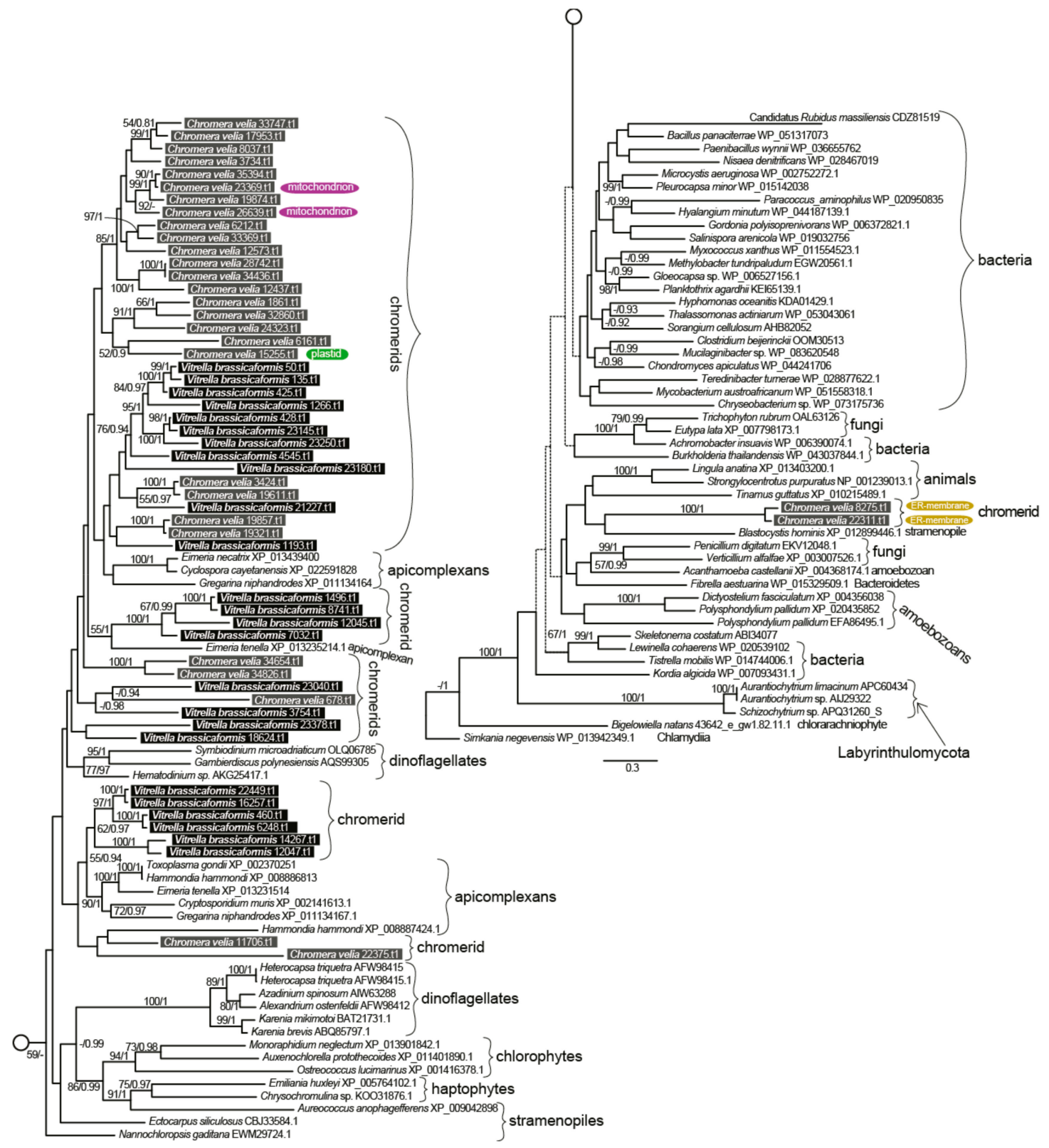

Figure 4. The detailed phylogeny of ketoacyl synthase domains of type I fatty acid synthases and polyketide synthases. Bootstrap for 1000 repetitions and Bayesian pp values (BS1000/1M, CAT/LG models) are displayed above the branches. Predicted subcellular locations other than cytosolic are shown.

\subsection{Elongation}

The repertoire of FA elongases (Tables 1-5) differs between the two chromerids, even though their detected FA spectra are highly similar (Figure 5). The class-specific sequence motifs [52] and phylogenetic signal in desaturase domains allowed us to recognize two major functional classes (Figure 6):

(i) Elongases specific for the saturated and monounsaturated fatty acids (S/MUFAs), forming four different orthologous groups. The total number of genes in each species is four, but their phylogenetic distribution within the tree and their predicted intracellular locations appeared to be species-specific. The first group predicted as cytosolic in location and counting one gene per species resulted to branch with stramenopiles. The second distant clade, putatively localized to the ER membrane, was found to be composed of $V$. brassicaformis (three genes) and $C$. velia (two genes). The remaining two S/MUFA 
elongases of $C$. velia and $V$. brassicaformis share origin with chlorophytes and plants at the very base of the S/MUFA group. A single distinct elongase of $C$. velia was found to form a sister long branch to haptophytes.

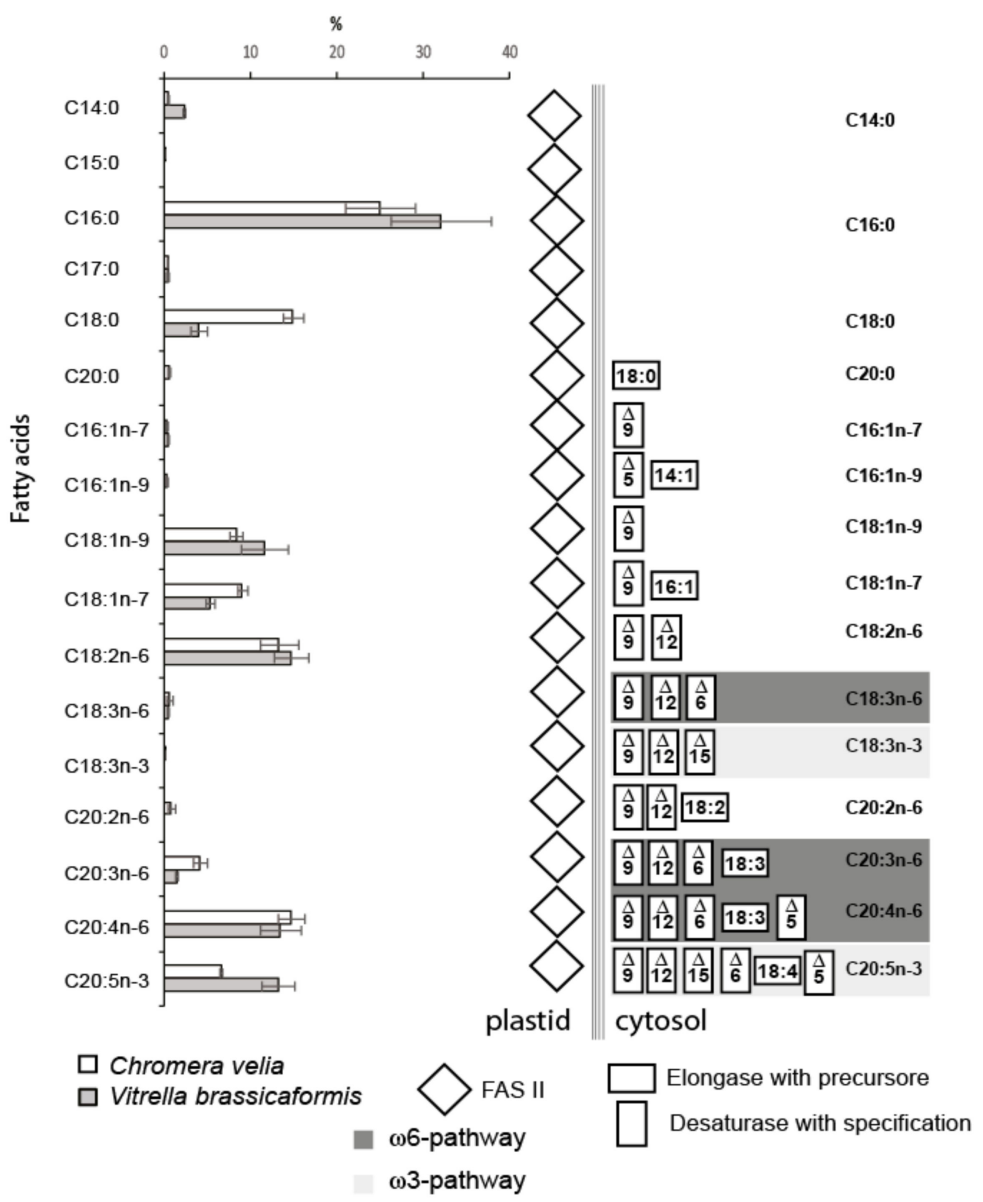

Figure 5. The fatty acid composition of both algae obtained by GC/FID (Chromera velia $350 \pm 38 \mathrm{mg} / \mathrm{g}$ and Vitrella brassicaformis $4 \pm 0.8 \mathrm{mg} / \mathrm{g}$ dry mass) and enzymatic repertoire for particular fatty acid synthesis and modification $(n=5)$. The concentrations of FAs are displayed in percentages with standard deviations. C. velia tends to accumulate storage lipids and contains approximately 100 -fold higher FAs concentrations than $V$. brassicaformis. The absolute concentrations of individual fatty acids can be calculated from absolute FAs concentrations values.

(ii) Elongases specific for poly-unsaturated FAs (PUFA). A single gene coding for a cytosolic elongase (Cvel_1604.t1) of PUFA was found in C. velia, whereas V. brassicaformis appeared to encode three paralogous enzymes putatively located in the ER membrane (Tables 1-5). The single cytosolic PUFA desaturase of $C$. velia clusters with stramenopiles, while the three ER-located elongases from $V$. brassicaformis branch together with apicomplexans (Figure 6). 


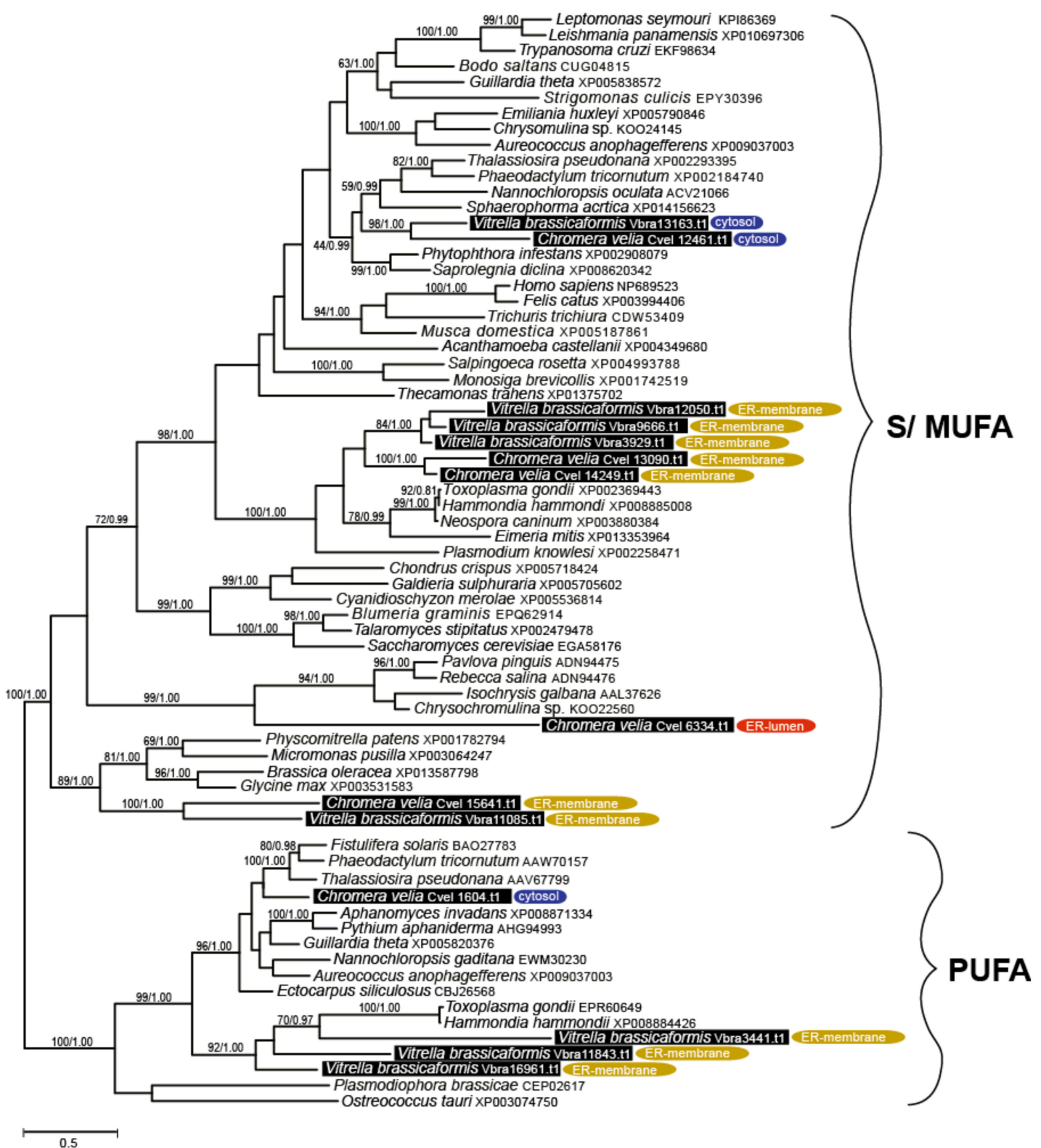

Figure 6. Bayesian phylogenetic tree inferred from elongase domains (70 taxa, 188 amino acid residues). Numbers above the branches display ML bootstrap/Bayesian PP support (BS1000/3M, CAT/LG). Predicted subcellular locations are displayed.

\subsection{Desaturation}

The desaturation of FAs is accomplished by desaturases of four different major classes defined by the specific products of their action. In both chromerid genomes, we identified desaturases of all functional classes. Most of the genes appeared to have no detectable $\mathrm{N}$-terminal-targeting pre-sequence (Tables 1-5), with omega desaturases making an exception (Tables 1-5). Therefore, we presume that most desaturation processes likely take place in the cytosol. To distinguish the main functional enzyme groups, we constructed a maximum likelihood phylogeny (Figure 7) based on the dataset of desaturase domains from various organisms previously published by Gostincar et al. [53]. Using this initial phylogeny-based sorting, we built more detailed phylogenies focused on particular desaturase classes (Figures S1-S3). 


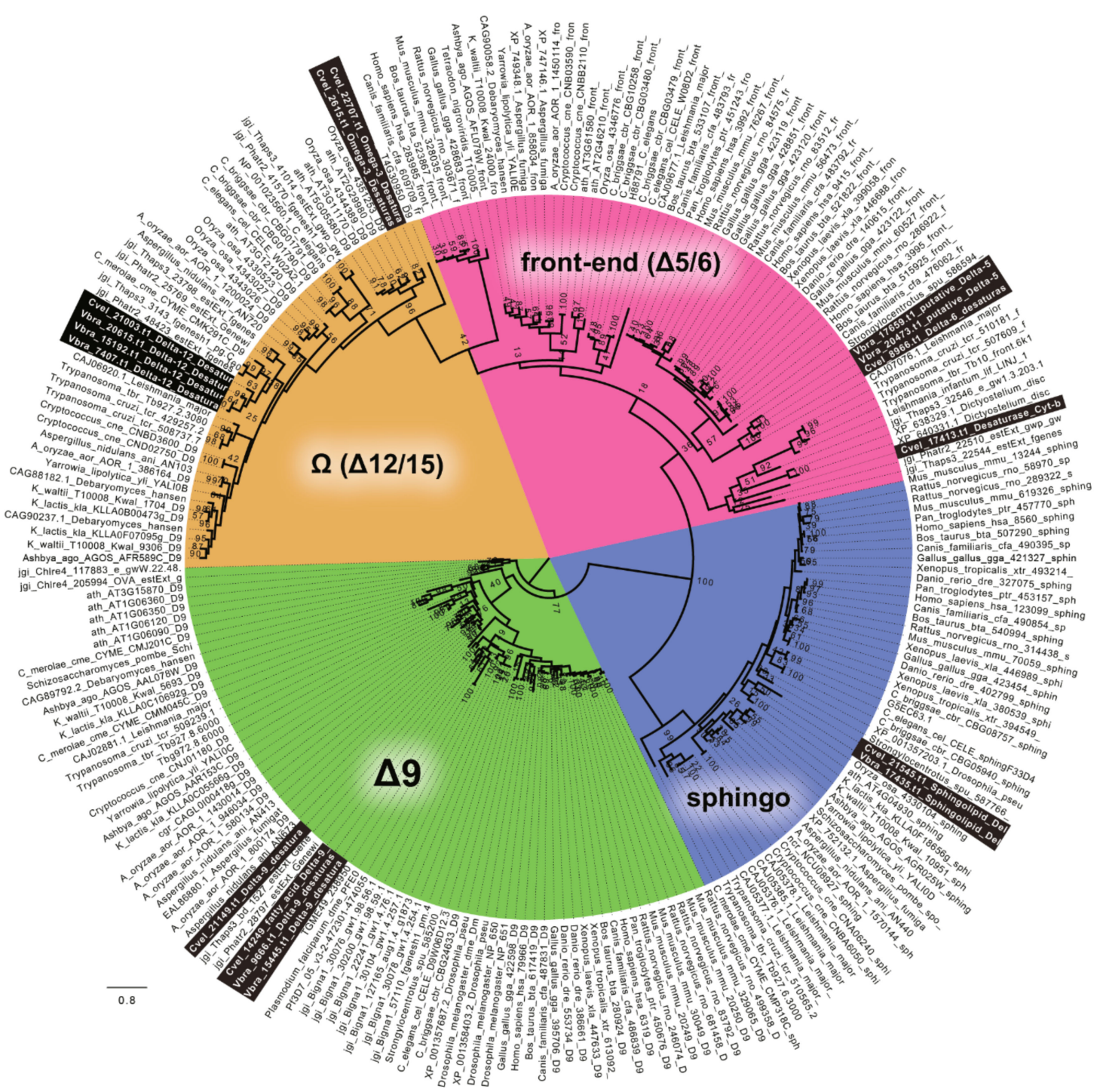

Figure 7. The RaxML tree resolving major functional classes of FA desaturases within various genomes, as described by Gostincar 2010 [50]. The chromerid genomes contain all types of desaturases. Detailed phylogenies of particular classes are displayed below.

Sphingolipid desaturases were not an object of this study. However, they were included in the phylogeny (Figure 7) to test the completeness of the dataset. Regarding the evolution of desaturases, $\Delta-9$ desaturases are generally considered as the ancestral group. The $\Delta-9$ class is represented by two genes in both C. velia and V. brassicaformis. The likelihood tree (Figure S1) suggested their origin within the SAR clade (Stramenopila + Alveolata + Rhizaria). The highly divergent gene Cvel_21149.t1 forms the long branch far distant from the SAR group or any sequence data deposited in databases. The prediction suggests a highly possible plastid targeting of Cvel_21149.t1 (Tables 1-5).

The $\Delta-5 / 6$ (front-end) enzyme class was found to be represented by two enzymes in each chromerid genome. The phylogenetic position of Cvel_17413.t1 is not fully resolved, likely due to high sequence divergence. The remaining genes form $C$. velia (1) and V. brassicaformis (2) coding for $\Delta-5 / 6$ (front-end) desaturases constitute a monophyletic sister group to their chlorophyte counterparts, branching in the proximity of perkinsids, rhizarian, haptophyte, kinetoplastids, stramenopiles, and choanoflagellate in the well-supported eukaryotic clade. TargetP predicted Vbra_20473.t1 as mitochondrially located (Figure 8, Figure S2). 


\begin{tabular}{|c|c|c|c|c|c|c|c|c|}
\hline Fatty acid & C.velia & V. brassicaformis & P. falciparum 1 & T.gondii ${ }^{2}$ & C. parvum ${ }^{3}$ & Symbiodinium sp. & P. tricomutum 5 & C. reinhardtii ${ }^{\circ}$ \\
\hline C14:0 & 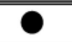 & 0 & 0 & 0 & 0 & 0 & $\overline{0}$ & 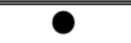 \\
\hline C16:0 & D & 0 & 0 & 0 & 0 & 0 & 0 & D \\
\hline C18:0 & 0 & 0 & - & 0 & 0 & 0 & 0 & D \\
\hline C20:0 & 0 & $\mathbf{x}$ & 0 & 0 & 0 & $x$ & $\mathbf{x}$ & - \\
\hline C22:0 & $x$ & $\mathbf{x}$ & $\bullet$ & 0 & 0 & $x$ & 0 & 0 \\
\hline C24:0 & $x$ & $x$ & - & 0 & 0 & $x$ & $x$ & 0 \\
\hline C16:1 & D & 0 & 0 & - & 0 & 0 & D & D \\
\hline C18:1 & - & ○ & - & - & 0 & 0 & 0 & ○ \\
\hline C20:1 & $x$ & $x$ & 0 & 0 & 0 & $x$ & $x$ & $x$ \\
\hline C22:1 & $x$ & $x$ & 0 & 0 & $x$ & 0 & $x$ & $x$ \\
\hline C24:1 & $\mathbf{x}$ & $\mathbf{x}$ & 0 & 0 & $x$ & $x$ & $\mathbf{x}$ & $\mathbf{x}$ \\
\hline C26:1 & $x$ & $x$ & $x$ & 0 & $x$ & $x$ & $x$ & $x$ \\
\hline C16:2 & $\mathbf{x}$ & $x$ & $x$ & $\mathbf{x}$ & $\mathbf{x}$ & $x$ & 0 & 0 \\
\hline C18:2 & 0 & 0 & 0 & $x$ & 0 & 0 & 0 & D \\
\hline $\mathrm{C} 20: 2$ & 0 & $x$ & $\mathbf{x}$ & $\mathbf{x}$ & $x$ & $x$ & $x$ & $x$ \\
\hline C16:3 & $x$ & $\mathbf{x}$ & $\mathbf{x}$ & $x$ & $x$ & $x$ & 0 & 0 \\
\hline C18:3 & D & 0 & $x$ & $x$ & 0 & $x$ & 0 & D \\
\hline C20:3 & 0 & 0 & 0 & $x$ & $\mathbf{x}$ & $x$ & 0 & $\mathbf{x}$ \\
\hline $\mathrm{C} 16: 4$ & $\mathbf{x}$ & $\mathbf{x}$ & $x$ & $\mathbf{x}$ & $x$ & $x$ & 0 & 0 \\
\hline C18:4 & $x$ & $x$ & $x$ & $x$ & $x$ & $x$ & 0 & 0 \\
\hline C20:4 & 0 & 0 & 0 & $x$ & 0 & $x$ & 0 & $x$ \\
\hline C20:5 & 0 & 0 & $x$ & $\mathbf{x}$ & $x$ & 0 & 0 & $x$ \\
\hline C22:6 & $x$ & $x$ & 0 & $x$ & $x$ & 0 & 0 & $x$ \\
\hline
\end{tabular}

$\mathbf{X}$ absent or not detected $\bigcirc$ synthetized $\bigcirc$ obtained from host $\bigcirc$ found in traces $\bigcirc$ nitrogen depletion $\quad \square$ increased under nitrogen depletion

Figure 8. Comparison of the fatty acid profile of particular organisms and the origin of particular fatty acids. Liver-stage of Plasmodium, intracellular stages of Toxoplasma gondii (1) Botté et al., 2013 [54], (2) Ramakrishnan et al., 2012, 2015 [52,55], (3) Mitschler et al., 1994 [56], (4) Weng at al., 2014 [57], (5) Siron et al., 1989; Popko et al., 2016 [58,59], (6) James et al., 2011; Puzanskiy et al., 2015 [60,61]. The position of the last double bond was not specified in some mentioned publications; therefore, it is not included in the table.

The $\Delta 12$-desaturase group found in C. velia forms two distinct clades: the first clade consists of two genes from C. velia, branching with plants and cyanobacteria, suggesting its plastid origin. The second clade is composed of one desaturase from C. velia together with three genes from $V$. brassicaformis and branches between stramenopiles and ciliates, within a major clade composed mainly of SAR group members, haptophytes, and cryptophytes (Figure S3). The N-terminal pre-sequence detected in the desaturase Cvel_22707.t1 sharing its origin with plants displays a bi-partite architecture characteristic for targeting complex plastids (Tables 1-5). The desaturase Vbra_7407.t1 shows only a hint of mitochondrial transit peptide, with low probability and reliability for the prediction. Thus, we considered this structure to be an artifact and this whole clade of chromerid desaturases as cytosolic.

\subsection{Fatty Acid Profile}

The list of all FAs detected in chromerids and their abundances are shown in Figure 5. The outcome of the described complex enzymatic machinery is a broad spectrum of FAs that are variable in abundance, number of carbons, number and position of double bonds. C. velia and V. brassicaformis have a high content of palmitic acid (C16:0), covering 25\% and 33\% of all FAs in the two algae. The FA profiles of chromerids are very similar to each other, with two exceptions: the occurrence of stearic acid (C18:0) in $C$. velia is three times higher than in $V$. brassicaformis, and $V$. brassicaformis contains two times more eicosapentaenoic acid (C20:5n-3) (EPA) than C. velia. The detected FAs (Figure 8, Figure 5) indicate the presence of both PUFA pathways $\omega 6$ (animals) and $\omega 3$ (algae), resulting in a chain of 20 carbon units with five double bonds (EPA). However, the last enzyme of the $\omega 6$ pathway, $\Delta-17$ desaturase, 
is missing in both algae in correlation with their high content of arachidonic acid (C20:4n-6). It seems that $V$. brassicaformis engages the $\omega 3$ pathway preferentially, unlike $C$. velia, which employs both pathways at a similar level (Figure 5, Figure S4a,b). The precursors of both pathways occur at very low levels or below the limit of detection (e.g., C18:3n-6, C18:3n-3, and C18:4n-3). The presence of C20:2n-6 among FAs of $C$. velia indicates an alternative $\omega 6$ pathway (Figure 3, Figure S4a). In contrast, C20:2n-6 is not present in V. brassicaformis, with no obvious alternative $\omega 6$ pathway (Figure S4b). The high variability of FAs detected in chromerids is also highlighted by the presence of oleic FA (C18:1n-9) and cis-vaccenic acid (C18:1n-7). These two FAs are based on palmitic acid, modified by only two enzymes, $\Delta-9$ desaturase and elongase. C18:1n-9 is synthesized by elongation of palmitic acid (C16:0) to stearic acid (C18:0) and subsequent desaturation at the 9th carbon position, in contrast to the synthesis of cis-vaccenic acid, which begins with the desaturation of palmitic acid at the 9 th carbon position followed by elongation, resulting in a shift of the double bond to the 7th position (for details, see Figure S4a,b).

After 20 days of triclosan treatment, all cultures treated with a concentration higher than $40 \mu \mathrm{M}$ stopped growing (Figure S5), bleached, and died. Cells treated with solvent control (DMSO) were not affected (data not shown). At the concentration of $40 \mu \mathrm{M}$, triclosan drastically inhibited the production of unsaturated FAs and slightly affected the abundance of saturated FAs. When the inhibitor concentration was increased up to $330 \mu \mathrm{M}$, we observed the vanishing of unsaturated FAs and a dramatic downregulation of C16:0. No such drastic decrease was observed in the production of C18:0 (Figure S6). Even a slightly higher concentration of triclosan in the medium caused a fatal collapse of the whole membrane system, as seen by transmission electron microscopy (Figure S7a,b).

Our experiment with nitrogen repletion and depletion showed changes in the lipid profiles of C. velia: FAs up to C18 increased in abundance by approximately $14 \%$ during nitrogen depletion, while FAs longer than C18 were more abundant during nitrogen repletion (Figure S8). The modulation of the FA profile by environmental conditions was also described [62].

\section{Discussion}

Both types of FAS have been detected in chromerids, T. gondii, and other coccidians (Figure 9). Type I FAS enzymes are preserved mainly in organisms that have lost their plastids, such as Cryptosporidium, or in those that never had plastids. It is possible that in plastid-bearing organisms, the rarely present cytosolic FAS-I is employed in the synthesis of some special FAs; however, it is more likely that FAS-I multi-modular enzymes in chromerids and coccidia are involved in the elongation of FAs rather than in de novo synthesis, as suggested for FAS-I enzyme in C. parvum. It was nicely shown that the multi-modular FAS-I enzyme from this plastid-lacking intestinal apicomplexan parasite is responsible for the elongation of short fatty acids scavenged from the host [33]. Since the acetyl-CoA (coenzyme A) carboxylase (ACC) from C. parvum, which is used for elongation and not FA de novo synthesis branches together with the cytosolic ACCs from T. gondii and chromerids (Figure S9), they can hypothetically do the same job. Although chromerids, coccidians, and Cryptosporidium are known to encode numerous FAS-I-like multi-modular enzymes in their genomes, only some of them are believed to be involved in FAS metabolism. Others more likely represent evolutionary related genes coding for type I polyketide synthases (PKS-I) responsible for the synthesis of polyketides, secondary metabolites known to act as antimicrobial compounds and toxins, such as cyanobacterial toxins, dinoflagellate red-tide toxins (e.g., brevetoxin), mycotoxins (e.g., aflatoxin), and even some compounds used as antibiotics (geldanamycin, doxycycline, and others) [51]. All these enzymes contain at least one incomplete module lacking an acyltransferase (AT) domain, with the absence of this domain being considered as a hallmark of most algal type I PKSs [25]. Furthermore, one and two multi-modular FAS-like enzymes in C. velia and $V$. brassicaformis, respectively, contain additional methyltransferase (MT) domains, which is also typical for PKSs [63]. 


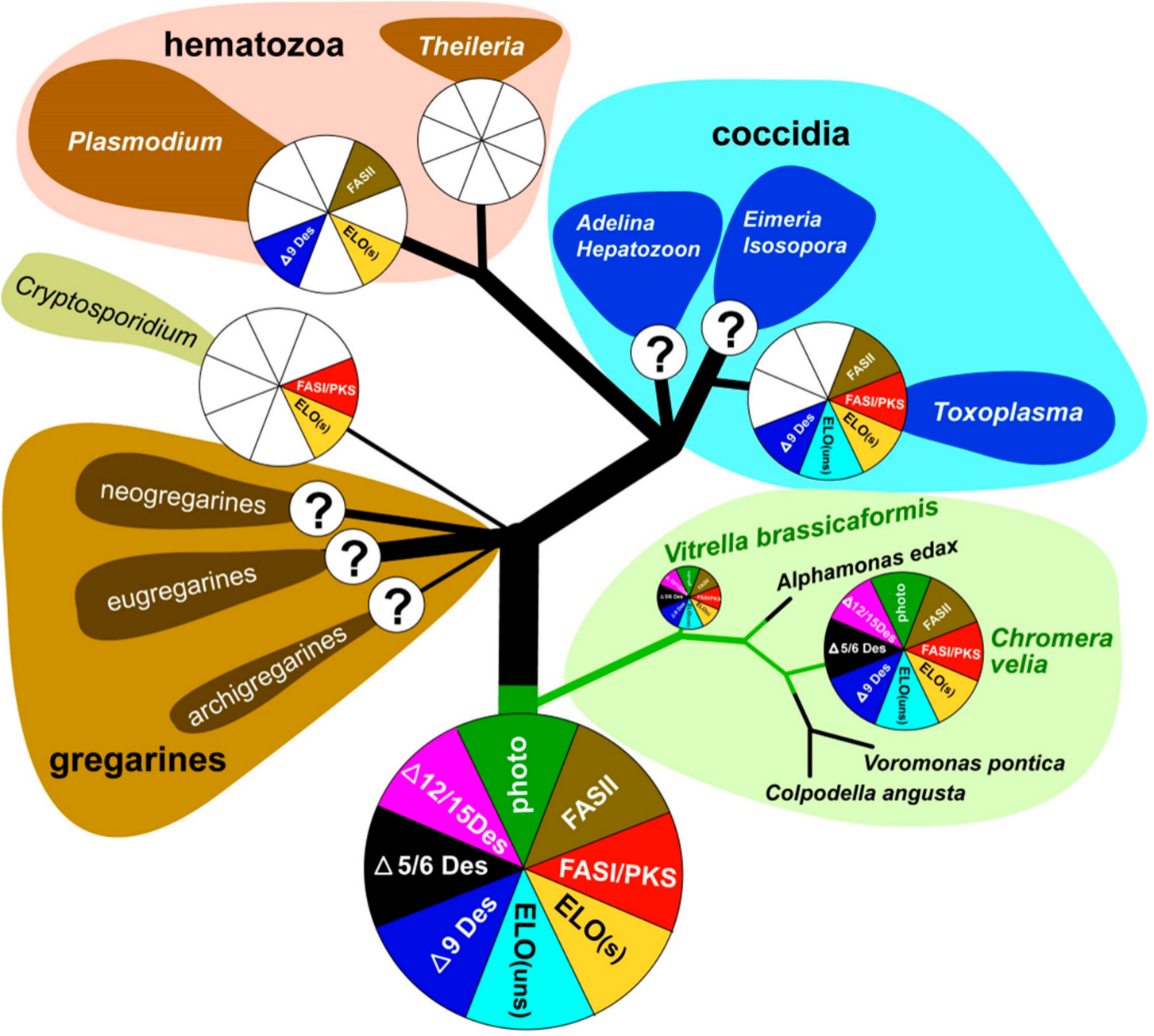

Figure 9. Overview of FAS evolution in chromerids and apicomplexan parasites.

The coccidian T. gondii contains at least one FAS-I enzyme with four complete modules $(9940$ amino acids) [57] and PKS-I enzymes [22]. C. parvum contains, in addition to the FAS-I multi-modular gene with three modules, also a PKS gene, which consists of seven modules containing 13,411 amino acids. The exclusive feature of all FAS-I and PKS-I in apicomplexans is likely the formation of highly resilient oocysts (Cryptosporidium, Eimeria, Toxoplasma) [30]. Since these enzymes have affinity to similar substrates, it is speculated that FAS-I, together with multi-domain PKSs, collaborate to produce special lipids constituting the oocyst membrane. This cooperation between FAS-I and PKS-I has also been reported in certain dinoflagellates, fungi, and bacteria [22]. A durable cell wall is also characteristic of certain life stages of $C$. velia and $V$. brassicaformis [1,2]. Although most multi-domain FAS-I/PKS-I enzymes in chromerids show sequence structures typical for PKS rather than FAS-I, both algae have two different ACCases (Figures S9 and S10). The first one is related to the plastid originated and located ACCases, similar to those of other apicomplexans, dinoflagellates, cryptophytes, haptophytes, and chlorophytes. The second one, which is likely cytosolic, branches with that of T. gondii and C. parvum. Since it was proposed that cytosolic acetyl CoA carboxylase could be a source of malonyl-CoA for the elongation of fatty acids [22,64], FAS-I in apicomplexans may be involved in elongation. As canonical elongases are also present in chromerid genomes, FAS-I multi-modular enzymes can be involved in FA elongation in specific life stages, like in T. gondii [65] and C. parvum [33].

As mentioned previously [13,58], chromerids are equipped with cytosolic FAS-I multi-modular enzymes originating from the eukaryotic heterotrophic host (exosymbiont) and with plastid-located FAS-II, which was acquired during the complex endosymbiotic event from the endosymbiont 
(Tables 1-5). This pathway arrangement correlates with the elevated expression of plastidial ACCase during nitrogen depletion, which has been described in C. velia, and in the haptophyte Isochrysis galbana [64]. The tendency of chromerids to switch metabolism towards storing energy in the form of lipids, which is enhanced in nitrogen-poor media, is shared with other algae like the symbiotic dinoflagellate Symbiodinium spp. [59], the eustigmatophyte Nannochloropsis gaditana [66], the diatom Phaeodactyllum tricornutum [60,61,67], the rhodophyte Cyanidioschyzon merolae, and the chlorophytes Chlorella vulgaris [68] and Chlamydomonas reinhardtii [55,69,70]. The complete FAS-II apicoplast-located system was recorded in T. gondii, P. falciparum, and E. tenella [22,29,54,71-75], and all these apicomplexans use it for the de novo synthesis of saturated FAs $[20,29,57,76]$. The apicomplexan minimalist Cryptosporidium does not possess the apicoplast, consequently lacks the FAS-II pathway, and fully depends on FAs scavenged from the host and in-house elongation [33]. The blood parasites Babesia and Theileria completely lack FAS and modification enzymes, despite the presence of the apicoplast in their cells [20,22,33] (Figure 9). Importantly, the FAS-II pathway was found in all organisms bearing red algal-derived plastids [23]. Based on experiments with the reportedly selective inhibitor of FAS-II triclosan [77], it was initially suggested that it is the FAS-I system which is responsible for FA de novo synthesis in C. velia, while FAS-II is involved in elongation and desaturation of primary FAs to final PUFAs arising from both $\omega-3$ and $\omega-6$ pathways [13]. A similar FA biosynthetic and modulatory system was initially proposed for T. gondii, again partly based on experiments with triclosan and other selective inhibitors. Mazumdar and Striepen [30] deduced that T. gondii employs FAS-I in the de novo synthesis of FAs and FAS-II in lipoic acid synthesis. However, experiments performed in the absence of selective inhibitors finally suggested that the de novo synthesis of FAs in T. gondii is maintained exclusively by the apicoplast-located type II FAS pathway [54,57]. After several of our experiments with triclosan and considering the above-mentioned published results, we doubt its inhibitory specificity. The target of triclosan is supposed to be the enoyl reductase domain, which plays an indispensable role in both FAS-I and FAS-II systems. Thus, the inhibition should affect both types of synthesis [78]. Since the enzymatic steps involved in the elongation process are like those of FAS-I and II, but the growing chain is held by CoA instead of ACP [30], we hypothesize that lower concentrations of triclosan mainly affect cytosolic enoyl reductases, while the plastidial ones are likely protected by multiple plastid membranes. It seems that plastid membrane protection is not efficient at triclosan concentrations of $80 \mu \mathrm{M}$ and higher. A fatal collapse of the whole membrane system, which is apparent in the TEM pictures (Figure S6), could be induced by the inhibition of plastidial enoyl reductase. We assume that the specificity of triclosan is at least questionable and that this compound non-specifically inhibits elongases and desaturases in the cytosol and endoplasmic reticulum rather than FA synthesis in the plastid. Therefore, we propose that the de novo synthesis of FAs occurs in the plastid using a prokaryotic type of fatty acid synthesis, like in other phototrophic eukaryotes. The FAS-II pathway was found responsible for FA synthesis in all organisms bearing red algal-derived plastids [23]. It also appears reasonable that one of the most energetically expensive processes, for which also reduction equivalents are needed, is associated with the plastid, the main 'power station' and producer of reduction equivalents of the phototrophic cell. Taking all the above-mentioned facts into account, we propose that chromerids synthesize at least the major pool of fatty acids in the plastid using the FAS-II system.

Sequence identification of the enzymes involved in FAS and their localization results (Tables 1-5) together with GC/FID analysis of FAs (Figure 5) provided enough information for proposing a model for FA biosynthesis, elongation, and desaturation in chromerids (Figure S4a,b). The primary sources of energy and carbons for the synthesis of FAs in chromerids are photosynthesis and the Calvin cycle, as described in plants [56,79]. Based on our findings, we suggest that the plastid-located FAS-II system de novo synthesizes short saturated FAs-mainly C16:0, followed by C18:0 and C14:0 acids-and exports them to the cytosol in the form of FAs or associated with ACP. FA-ACP complexes are then modified by the set of desaturases and elongases. Most enzymes participating in the final steps of FA synthesis are either located in the cytosol or associated with the membrane of the endoplasmic reticulum, with the 
exception of one plastid-targeted $\Delta 9$ desaturase (Cvel_21149), one plastid-targeted $\omega$-desaturase (Cvel_22707), and one front-end desaturase (Vbra 20473), likely located in the mitochondrion (Tables 1-5). Plastid-targeted $\Delta 9$ and $\omega$-desaturases were also described in plants [24] and are the only desaturases retained in Plasmodium lacking any traces of FAS-I system (Figure 9). It should also be pointed out that chromerids do not produce C16 fatty acids with more than one double bond. Such poly-unsaturated C16 fatty acids are common in a broad spectrum of non-chromerid algae; for example, the diatom P. tricornutum and the green alga C. reinhardtii contain these FAs even with four double bonds in the 16 carbons chain (Figure 8). The presence of an alternative pathway to produce EPA in C. velia is very common and has been previously reported in the chlorophyte Pariechloris incista [80]. Furthermore, the diatom P. tricornutum has three [81], and Euglena gracilis has four different pathways to produce EPA [82]. The absence of any alternative EPA synthesis pathway in $V$. brassicaformis is even more interesting (Figure S4b). Chromerids lack long FAs (longer than C20), like other mentioned eukaryotic phototrophs, except for P. tricornutum, containing polyunsaturated FAs (22:6) (Figure 8).

Chromerids encode a broad spectrum of enzymes involved in the synthesis of short-chain saturated FAs and their derivatives. The evolutionary diagram in Figure 9 displays differences between chromerids and apicomplexans, which have completely lost $\Delta 5 / 6$ and $\Delta 12 / 15$ desaturases essential for the biosynthesis of PUFAs. However, previous works dealing with fatty acid determination showed the occurrence of PUFA in P. falciparum [76] and C. parvum [83] (for details, see Figure 8.). The presence of PUFA in these two parasites can be explained by their salvage from the host. The only known apicomplexans completely lacking the apicoplast are eugregarines (like Gregarina niphandrodes) [84] and members of the genus Cryptosporidium $[85,86]$. Due to the loss of the complete plastidial FAS II pathway and all desaturases and elongases, $C$. parvum displays the most reduced enzymatic equipment for FA synthesis and modification among apicomplexans. Like C. velia, T. gondii and E. tenella have retained the majority of the enzymatic equipment, except for $\Delta 5 / 6$ and $\Delta 12 / 15$ desaturases. While $T$. gondii uses three elongases and only a single $\Delta 9$ desaturase [54], C. velia and $V$. brassicaformis encode six and eight elongases, respectively, and two $\Delta 9$ desaturases. The loss of photosynthesis and switch to parasitism have been accompanied by losses of enzymes involved in FA synthesis in a lineage-specific pattern. The ancestral state was most likely equipped as the typical photosynthetic primary producer.

Cytosolic S/MUFA elongases branch along with stramenopiles (Figure 4), suggesting the possible transfer from a hypothetical tertiary stramenopile endosymbiont linked to the origin of the chromerid plastid $[2,4,87,88]$. The group of S/MUFA elongases branching along with apicomplexans reflects the common origin of the chromerids and apicomplexans in the frame of the myzozoan lineage. A single gene of C. velia (Cvel_6334.t1) constitutes a long branch, sister to haptophytes. Similarly, a single PUFA elongase found in C. velia (Cvel_1604.t1) is related to stramenopiles, in contrast to three genes from $V$. brassicaformis, with apicomplexan relatives. Long branching and orphan phylogenetic positions are common phenomena for genes of $C$. velia. It appears that the alga retained genes of ancient origin, acting as a collector of genetic fossils.

\section{Conclusions}

Sequence identification of particular enzymes involved in FA synthesis and their predicted localization (Tables 1-5) together with GC analysis of particular FAs (Figure 5) provided enough information for proposing a system of fatty acid biosynthesis, elongation, and desaturation in chromerids (Figure S4). The primary sources of energy and carbons for the synthesis of fatty acids in chromerids are photosynthesis and the Calvin cycle, as described in plants [56,79]. We propose that the FAS II system localized in the plastid produces de novo short saturated fatty acids-mainly C16:0 (palmitic acid), followed by C18:0 (stearic acid) and C14:0 (myristic acid)-and exports them to the cytosol as fatty acids or in association with ACP. Fatty acids with ACP are then modified by the set of desaturases and elongases. Most enzymes participating in the final steps of fatty acid synthesis are either localized in the cytosol or associated with the membrane of the endoplasmic reticulum, with the exclusion of one $\Delta 9$ desaturase (Cvel21149), one $\omega$-desaturase (Cvel22707), which are 
putatively localized in the plastid (Tables 1-5), as described in plants [24], and one front-end desaturase (Vbra 20473) probably localized in the mitochondrion.

Supplementary Materials: The following are available online at http://www.mdpi.com/2218-273X/10/8/1102/ s1: Figure S1. The $\Delta-9$ desaturases Likelihood/Bayesian tree. Figure S2. The $\Delta 5 / 6$ (front-end) desaturase Likelihood/Bayesian tree. Figure S3. The omega $(\Delta 12 / 15)$ desaturase Likelihood/Bayesian tree. Figure S4. The model of fatty acid biosynthesis in Chromera velia (A) and in Vitrella brassicaformis (B). Figure S5. Growing curves of Chromera velia treated by the various concentration of triclosan recorded by the Tecan instrument $(n=5)$. Figure S6. Spectra of fatty acid composition changes after triclosan treatment obtained by GC/FID. Figure S7 Transmission electron microscopy of $C$. velia grown in standard $f / 2$ medium (A) and grown in $f / 2$ medium with $333 \mu \mathrm{M}$ triclosan (B), where the cell is deformed and the inner structure is collapsed. LD-lipid droplets; $\mathrm{N}$-nucleus; P-plastid. Figure S8. The ratio of fatty acids shorter than C18 and C18 (blue) and longer than C18 (orange) in C.velia during nitrogen deprivation and repletion $(n=5)$. Figure S9. Maximum-likelihood phylogenetic tree of acetyl-CoA decarboxylases. Figure S10. The alignment of ACCases of chromerids, showing the first 408 amino acid residues, with the N-terminal motif similarity shared between plastid-targeted gene variants.

Author Contributions: M.O. conceived the study. A.T. performed experiments, interpreted GC/FID data, proposed the model of fatty acid biosynthesis. J.M. performed genomic searches and phylogenetic analyses and interpreted the results. I.S. extracted lipids, prepared samples for derivatization, and performed the GC/FID analysis. M.V. prepared the T.E.M. samples. A.T., J.M., M.O., Z.F. and A.G. wrote the manuscript. MO obtained funding. All authors have read and agreed to the published version of the manuscript.

Funding: This research was funded by the Czech Science Foundation (project 18-13458S), Czech BioImaging (MEYS CR, LM2015062), Ministry of Education, Youth, and Sports of the Czech Republic-the CENAKVA project (LM2018099), CENAKAVA Center Development (CZ.1.05/2.1.00/19.0380), and ERDF/ESF Centre for research of pathogenicity and virulence of parasites (No.CZ.02.1.01/0.0/0.0/16_019/0000759).

Acknowledgments: We thank Geoffrey I. McFadden for helpful discussions.

Conflicts of Interest: The authors declare no conflict of interest

\section{References}

1. Moore, R.B.; Oborník, M.; Janouškovec, J.; Chrudimský, T.; Vancová, M.; Green, D.H.; Wright, S.W.; Davies, N.W.; Bolch, C.J.S.; Heimann, K.; et al. A photosynthetic alveolate closely related to apicomplexan parasites. Nature 2008, 451, 959-963. [CrossRef] [PubMed]

2. Oborník, M.; Modrý, D.; Lukeš, M.; Černotiková-Stř̌ibrná, E.; Cihlář, J.; Tesařová, M.; Kotabová, E.; Vancová, M.; Prášil, O.; Lukeš, J. Morphology, Ultrastructure and Life Cycle of Vitrella brassicaformis n. sp., n. gen., a Novel Chromerid from the Great Barrier Reef. Protist 2012, 163, 306-323. [CrossRef] [PubMed]

3. Oborník, M.; Janouškovec, J.; Chrudimský, T.; Lukeš, J. Evolution of the apicoplast and its hosts: From heterotrophy to autotrophy and back again. Int. J. Parasitol. 2009, 39, 1-12. [CrossRef] [PubMed]

4. Füssy, Z.; Oborník, M. Chromerids and Their Plastids. In Secondary Endosymbioses; Hirakawa, Y., Ed.; Academic Press Ltd-Elsevier Science Ltd.: London, UK, 2017; Volume 84, pp. 187-218.

5. Cumbo, V.R.; Baird, A.H.; Moore, R.B.; Negri, A.P.; Neilan, B.A.; Salih, A.; van Oppen, M.J.H.; Wang, Y.; Marquis, C.P. Chromera velia is Endosymbiotic in Larvae of the Reef Corals Acropora digitifera and A. tenuis. Protist 2013, 164, 237-244. [CrossRef]

6. Janouškovec, J.; Sobotka, R.; Lai, D.; Flegontov, P.; Koník, P.; Komenda, J.; Ali, S.; Prášil, O.; Pain, A.; Oborník, M.; et al. Split Photosystem Protein, Linear-Mapping Topology, and Growth of Structural Complexity in the Plastid Genome of Chromera velia. Mol. Biol. Evol. 2013, 30, 2447-2462. [CrossRef]

7. Okamoto, N.; McFadden, G.I. The mother of all parasites. Future Microbiol. 2008, 3, 391-395. [CrossRef]

8. Mohamed, A.R.; Cumbo, V.R.; Harii, S.; Shinzato, C.; Chan, C.X.; Ragan, M.A.; Satoh, N.; Ball, E.E.; Miller, D.J. Deciphering the nature of the coral-Chromera association. ISME J. 2018, 12, 776-790. [CrossRef]

9. Mathur, V.; del Campo, J.; Kolisko, M.; Keeling, P.J. Global diversity and distribution of close relatives of apicomplexan parasites. Environ. Microbiol. 2018, 20, 2824-2833. [CrossRef]

10. Janouškovec, J.; Tikhonenkov, D.V.; Burki, F.; Howe, A.T.; Kolisko, M.; Mylnikov, A.P.; Keeling, P.J. Factors mediating plastid dependency and the origins of parasitism in apicomplexans and their close relatives. Proc. Natl. Acad. Sci. USA 2015, 112, 10200-10207. [CrossRef]

11. Janouškovec, J.; Horák, A.; Oborník, M.; Lukeš, J.; Keeling, P.J. A common red algal origin of the apicomplexan, dinoflagellate, and heterokont plastids. Proc. Natl. Acad. Sci. USA 2010, 107, 10949-10954. [CrossRef] 
12. Kořený, L.; Sobotka, R.; Janouškovec, J.; Keeling, P.J.; Oborník, M. Tetrapyrrole Synthesis of Photosynthetic Chromerids Is Likely Homologous to the Unusual Pathway of Apicomplexan Parasites. Plant Cell 2011, 23, 3454-3462. [CrossRef] [PubMed]

13. Woo, Y.H.; Ansari, H.; Otto, T.D.; Klinger, C.M.; Kolisko, M.; Michálek, J.; Saxena, A.; Shanmugam, D.; Tayyrov, A.; Veluchamy, A.; et al. Chromerid genomes reveal the evolutionary path from photosynthetic algae to obligate intracellular parasites. Elife 2015, 4. [CrossRef]

14. Oborník, M. Endosymbiotic Evolution of Algae, Secondary Heterotrophy and Parasitism. Biomolecules 2019, 9. [CrossRef] [PubMed]

15. Flegontov, P.; Michálek, J.; Janouškovec, J.; Lai, D.H.; Jirků, M.; Hajdůšková, E.; Tomčala, A.; Otto, T.D.; Keeling, P.J.; Pain, A.; et al. Divergent Mitochondrial Respiratory Chains in Phototrophic Relatives of Apicomplexan Parasites. Mol. Biol. Evol. 2015, 32, 1115-1131. [CrossRef] [PubMed]

16. Oborník, M.; Vancová, M.; Lai, D.-H.; Janouškovec, J.; Keeling, P.J.; Lukeš, J. Morphology and Ultrastructure of Multiple Life Cycle Stages of the Photosynthetic Relative of Apicomplexa, Chromera velia. Protist 2011, 162, 115-130. [CrossRef] [PubMed]

17. Füssy, Z.; Masařova, P.; Kručinská, J.; Esson, H.J.; Oborník, M. Budding of the Alveolate Alga Vitrella brassicaformis Resembles Sexual and Asexual Processes in Apicomplexan Parasites. Protist 2017, 168, 80-91. [CrossRef] [PubMed]

18. Cavalier-Smith, T. Kingdom Chromista and its eight phyla: A new synthesis emphasising periplastid protein targeting, cyto-skeletal and periplastid evolution, and ancient divergences. Protoplasma 2018, 255, 297-357. [CrossRef]

19. Fahy, E.; Subramaniam, S.; Brown, H.A.; Glass, C.K.; Merrill, A.H.; Murphy, R.C.; Raetz, C.R.H.; Russell, D.W.; Seyama, Y.; Shaw, W.; et al. A comprehensive classification system for lipids. J. Lipid Res. 2005, 46, 839-861. [CrossRef]

20. Goodman, C.D.; McFadden, G.I. Fatty acid biosynthesis as a drug target in apicomplexan parasites. Curr. Drug Targets 2007, 8, 15-30. [CrossRef]

21. Chirala, S.S.; Wakil, S.J. Structure and function of animal fatty acid synthase. Lipids 2004, 39, 1045-1053. [CrossRef]

22. Ramakrishnan, S.; Serricchio, M.; Striepen, B.; Butikofer, P. Lipid synthesis in protozoan parasites: A comparison between kinetoplastids and apicomplexans. Prog. Lipid Res. 2013, 52, 488-512. [CrossRef] [PubMed]

23. Ryall, K.; Harper, J.T.; Keeling, P.J. Plastid-derived Type II fatty acid biosynthetic enzymes in chromists. Gene 2003, 313, 139-148. [CrossRef]

24. Schmid, K.M. Biochemistry of Lipids, Lipoproteins and Membranes; Elsevier B.V.: Amsterdam, The Netherlands, 2016; pp. 113-147.

25. Shelest, E.; Heimerl, N.; Fichtner, M.; Sasso, S. Multimodular type I polyketide synthases in algae evolve by module duplications and displacement of AT domains in trans. BMC Genom. 2015, 16. [CrossRef] [PubMed]

26. McFadden, G.I. Plastids and protein targeting. J. Eukaryot. Microbiol. 1999, 46, 339-346. [CrossRef]

27. Wilson, R.J.M. Plastid functions in the Apicomplexa. Protist 2004, 155, 11-12. [CrossRef]

28. Zhu, G. Current progress in the fatty acid metabolism in Cryptosporidium parvum. J. Eukaryot. Microbiol. 2004, 51, 381-388. [CrossRef]

29. Lu, J.Z.; Muench, S.P.; Allary, M.; Campbell, S.; Roberts, C.W.; Mui, E.; McLeod, R.L.; Rice, D.W.; Prigge, S.T. Type I and type II fatty acid biosynthesis in Eimeria tenella: Enoyl reductase activity and structure. Parasitology 2007, 134, 1949-1962. [CrossRef]

30. Mazumdar, J.; Striepen, B. Make it or take it: Fatty acid metabolism of Apicomplexan parasites. Eukaryot. Cell 2007, 6, 1727-1735. [CrossRef]

31. Sonda, S.; Hehl, A.B. Lipid biology of Apicomplexa: Perspectives for new drug targets, particularly for Toxoplasma gondii. Trends Parasitol. 2006, 22, 41-47. [CrossRef]

32. Salomaki, E.D.; Kolisko, M. There Is Treasure Everywhere: Reductive Plastid Evolution in Apicomplexa in Light of Their Close Relatives. Biomolecules 2019, 9. [CrossRef]

33. Zhu, G.; Li, Y.N.; Cai, X.M.; Millership, J.J.; Marchewka, M.J.; Keithly, J.S. Expression and functional characterization of a giant Type I fatty acid synthase (CpFAS1) gene from Cryptosporidium parvum. Mol. Biochem. Parasitol. 2004, 134, 127-135. [CrossRef] 
34. John, U.; Beszteri, B.; Derelle, E.; de Peer, Y.V.; Read, B.; Moreau, H.; Cembella, A. Novel insights into evolution of protistan polyketide synthases through phylogenomic analysis. Protist 2008, 159, 21-30. [CrossRef] [PubMed]

35. Heath, R.J.; Rubin, J.R.; Holland, D.R.; Zhang, E.L.; Snow, M.E.; Rock, C.O. Mechanism of triclosan inhibition of bacterial fatty acid synthesis. J. Biol. Chem. 1999, 274, 11110-11114. [CrossRef] [PubMed]

36. Katoh, K.; Misawa, K.; Kuma, K.; Miyata, T. MAFFT: A novel method for rapid multiple sequence alignment based on fast Fourier transform. Nucleic Acids Res. 2002, 30, 3059-3066. [CrossRef] [PubMed]

37. Kearse, M.; Moir, R.; Wilson, A.; Stones-Havas, S.; Cheung, M.; Sturrock, S.; Buxton, S.; Cooper, A.; Markowitz, S.; Duran, C.; et al. Geneious Basic: An integrated and extendable desktop software platform for the organization and analysis of sequence data. Bioinformatics 2012, 28, 1647-1649. [CrossRef] [PubMed]

38. Stamatakis, A. RAxML version 8: A tool for phylogenetic analysis and post-analysis of large phylogenies. Bioinformatics 2014, 30, 1312-1313. [CrossRef] [PubMed]

39. Huelsenbeck, J.P.; Bollback, J.P. Empirical and hierarchical Bayesian estimation of ancestral states. Syst. Biol. 2001, 50, 351-366. [CrossRef]

40. Ronquist, F.; Huelsenbeck, J.P. MrBayes 3: Bayesian phylogenetic inference under mixed models. Bioinformatics 2003, 19, 1572-1574. [CrossRef]

41. Emanuelsson, O.; Nielsen, H.; Brunak, S.; von Heijne, G. Predicting subcellular localization of proteins based on their N-terminal amino acid sequence. J. Mol. Biol. 2000, 300, 1005-1016. [CrossRef]

42. Nielsen, H.; Engelbrecht, J.; Brunak, S.; von Heijne, G. Identification of prokaryotic and eukaryotic signal peptides and prediction of their cleavage sites. Protein Eng. 1997, 10, 1-6. [CrossRef]

43. Bendtsen, J.D.; Nielsen, H.; von Heijne, G.; Brunak, S. Improved prediction of signal peptides: SignalP 3.0. J. Mol. Biol. 2004, 340, 783-795. [CrossRef] [PubMed]

44. Gruber, A.; Rocap, G.; Kroth, P.G.; Armbrust, E.V.; Mock, T. Plastid proteome prediction for diatoms and other algae with secondary plastids of the red lineage. Plant J. 2015, 81, 519-528. [CrossRef] [PubMed]

45. Miller, M.A.; Pfeiffer, W.; Schwartz, T. Creating the CIPRES Science Gateway for inference of large phylogenetic trees. In Proceedings of the 2010 Gateway Computing Environments Workshop (GCE), New Orleans, LA, USA, 14 November 2010; pp. 1-8.

46. Folch, J.; Lees, M.; Stanley, G.H.S. A simple method for the isolation and purification of total lipides from animal tissues. J. Biol. Chem. 1957, 226, 497-509. [PubMed]

47. Košt'ál, V.; Šimek, P. Changes in fatty acid composition of phospholipids and triacylglycerols after cold-acclimation of an aestivating insect prepupa. J. Comp. Physiol. B Biochem. Syst. Environ. Physiol. 1998, 168, 453-460. [CrossRef]

48. Tomčala, A.; Kyselová, V.; Schneedorferová, I.; Opekarová, I.; Moos, M.; Urajová, P.; Kručinská, J.; Oborník, M. Separation and identification of lipids in the photosynthetic cousins of Apicomplexa Chromera velia and Vitrella brassicaformis. J. Sep. Sci. 2017, 40, 3402-3413. [CrossRef] [PubMed]

49. Zahradníčková, H.; Tomčala, A.; Berková, P.; Schneedorferová, I.; Okrouhlík, J.; Šimek, P.; Hodková, M. Cost effective, robust, and reliable coupled separation techniques for the identification and quantification of phospholipids in complex biological matrices: Application to insects. J. Sep. Sci. 2014, 37, 2062-2068. [CrossRef]

50. Dahmen, J.L.; Khadka, M.; Dodson, V.J.; Leblond, J.D. Mono- and digalactosyldiacylglycerol composition of dinoflagellates. VI. Biochemical and genomic comparison of galactolipid biosynthesis between Chromera velia (Chromerida), a photosynthetic alveolate with red algal plastid ancestry, and the dinoflagellate, Lingulodinium polyedrum. Eur. J. Phycol. 2013, 48, 268-277. [CrossRef]

51. Jenke-Kodama, H.; Sandmann, A.; Muller, R.; Dittmann, E. Evolutionary implications of bacterial polyketide synthases. Mol. Biol. Evol. 2005, 22, 2027-2039. [CrossRef]

52. Hashimoto, K.; Yoshizawa, A.C.; Okuda, S.; Kuma, K.; Goto, S.; Kanehisa, M. The repertoire of desaturases and elongases reveals fatty acid variations in 56 eukaryotic genomes. J. Lipid Res. 2008, 49, 183-191. [CrossRef]

53. Gostincar, C.; Turk, M.; Gunde-Cimerman, N. The Evolution of Fatty Acid Desaturases and Cytochrome b5 in Eukaryotes. J. Membr. Biol. 2010, 233, 63-72. [CrossRef] 
54. Ramakrishnan, S.; Docampo, M.D.; MacRae, J.I.; Pujol, F.M.; Brooks, C.F.; van Dooren, G.G.; Hiltunen, J.K.; Kastaniotis, A.J.; McConville, M.J.; Striepen, B. Apicoplast and Endoplasmic Reticulum Cooperate in Fatty Acid Biosynthesis in Apicomplexan Parasite Toxoplasma gondii. J. Biol. Chem. 2012, 287, 4957-4971. [CrossRef]

55. James, G.O.; Hocart, C.H.; Hillier, W.; Chen, H.C.; Kordbacheh, F.; Price, G.D.; Djordjevic, M.A. Fatty acid profiling of Chlamydomonas reinhardtii under nitrogen deprivation. Bioresour. Technol. 2011, 102, 3343-3351. [CrossRef] [PubMed]

56. Rawsthorne, S. Carbon flux and fatty acid synthesis in plants. Prog. Lipid Res. 2002, 41, 182-196. [CrossRef]

57. Ramakrishnan, S.; Docampo, M.D.; MacRae, J.I.; Ralton, J.E.; Rupasinghe, T.; McConville, M.J.; Striepen, B. The intracellular parasite Toxoplasma gondii depends on the synthesis of long-chain and very long-chain unsaturated fatty acids not supplied by the host cell. Mol. Microbiol. 2015, 97, 64-76. [CrossRef] [PubMed]

58. Füssy, Z.; Faitova, T.; Oborník, M. Subcellular Compartments Interplay for Carbon and Nitrogen Allocation in Chromera velia and Vitrella brassicaformis. Genome Biol. Evol. 2019, 11, 1765-1779. [CrossRef]

59. Weng, L.C.; Pasaribu, B.; Lin, I.P.; Tsai, C.H.; Chen, C.S.; Jiang, P.L. Nitrogen Deprivation Induces Lipid Droplet Accumulation and Alters Fatty Acid Metabolism in Symbiotic Dinoflagellates Isolated from Aiptasia pulchella. Sci. Rep. 2014, 4. [CrossRef]

60. Siron, R.; Giusti, G.; Berland, B. Changes in the fatty-acid composition of Phaeodactylum tricornutum and Dunaliella tertiolecta during growth and under phosphorus deficiency. Mar. Ecol. Prog. Ser. 1989, 55, 95-100. [CrossRef]

61. Popko, J.; Herrfurth, C.; Feussner, K.; Ischebeck, T.; Iven, T.; Haslam, R.; Hamilton, M.; Sayanova, O.; Napier, J.; Khozin-Goldberg, I.; et al. Metabolome Analysis Reveals Betaine Lipids as Major Source for Triglyceride Formation, and the Accumulation of Sedoheptulose during Nitrogen-Starvation of Phaeodactylum tricornutum. PLoS ONE 2016, 11. [CrossRef]

62. Lukeš, M.; Giordano, M.; Prasil, O. The effect of environmental factors on fatty acid composition of Chromera velia (Chromeridae). J. Appl. Phycol. 2017, 29, 1791-1799. [CrossRef]

63. Skiba, M.A.; Sikkema, A.P.; Fiers, W.D.; Gerwick, W.H.; Sherman, D.H.; Aldrich, C.C.; Smith, J.L. Domain Organization and Active Site Architecture of a Polyketide Synthase C-methyltransferase. ACS Chem. Biol. 2016, 11, 3319-3327. [CrossRef]

64. Huerlimann, R.; Steinig, E.J.; Loxton, H.; Zenger, K.R.; Jerry, D.R.; Heimann, K. The effect of nitrogen limitation on acetyl-CoA carboxylase expression and fatty acid content in Chromera velia and Isochrysis aff. galbana (TISO). Gene 2014, 543, 204-211. [CrossRef]

65. Dubois, D.; Fernandes, F.; Amiar, S.; Dass, S.; Katris, N.J.; Botté, C.Y.; Yamaryo-Botté, Y. Toxoplasma gondii acetyl-CoA synthetase is involved in fatty acid elongation (of long fatty acid chains) during tachyzoite life stages. J. Lipid Res. 2018, 59, 994-1004. [CrossRef] [PubMed]

66. Simionato, D.; Block, M.A.; La Rocca, N.; Jouhet, J.; Marechal, E.; Finazzi, G.; Morosinotto, T. The Response of Nannochloropsis gaditana to Nitrogen Starvation Includes De Novo Biosynthesis of Triacylglycerols, a Decrease of Chloroplast Galactolipids, and Reorganization of the Photosynthetic Apparatus. Eukaryot. Cell 2013, 12, 665-676. [CrossRef]

67. Burrows, E.H.; Bennette, N.B.; Carrieri, D.; Dixon, J.L.; Brinker, A.; Frada, M.; Baldassano, S.N.; Falkowski, P.G.; Dismukes, G.C. Dynamics of Lipid Biosynthesis and Redistribution in the Marine Diatom Phaeodactylum tricornutum Under Nitrate Deprivation. Bioenergy Res. 2012, 5, 876-885. [CrossRef]

68. Stephenson, A.L.; Dennis, J.S.; Howe, C.J.; Scott, S.A.; Smith, A.G. Influence of nitrogen-limitation regime on the production by Chlorella vulgaris of lipids for biodiesel feedstocks. Biofuels 2010, 1, 47-58. [CrossRef]

69. Yang, D.W.; Song, D.H.; Kind, T.; Ma, Y.; Hoefkens, J.; Fiehn, O. Lipidomic Analysis of Chlamydomonas reinhardtii under Nitrogen and Sulfur Deprivation. PLoS ONE 2015, 10. [CrossRef] [PubMed]

70. Puzanskiy, R.K.; Shavarda, A.L.; Tarakhovskaya, E.R.; Shishova, M.F. Analysis of Metabolic Profile of Chlamydomonas reinhardtii Cultivated under Autotrophic Conditions. Appl. Biochem. Microbiol. 2015, 51, 83-94. [CrossRef]

71. Bisanz, C.; Bastien, O.; Grando, D.; Jouhet, J.; Marechal, E.; Cesbron-Delauw, M.F. Toxoplasma gondii acyl-lipid metabolism: De novo synthesis from apicoplast-generated fatty acids versus scavenging of host cell precursors. Biochem. J. 2006, 394, 197-205. [CrossRef] 
72. Lack, G.; Homberger-Zizzari, E.; Folkers, G.; Scapozza, L.; Perozzo, R. Recombinant expression and biochemical characterization of the unique elongating beta-ketoacyl-acyl carrier protein synthase involved in fatty acid biosynthesis of Plasmodium falciparum using natural and artificial substrates. J. Biol. Chem. 2006, 281, 9538-9546. [CrossRef]

73. Sharma, S.K.; Kapoor, M.; Ramya, T.N.C.; Kumar, S.; Kumar, G.; Modak, R.; Sharma, S.; Surolia, N.; Surolia, A. Identification, characterization, and inhibition of Plasmodium falciparum beta-hydroxyacyl-acyl carrier protein dehydratase (FabZ). J. Biol. Chem. 2003, 278, 45661-45671. [CrossRef]

74. Surolia, N.; Surolia, A. Triclosan offers protection against blood stages of malaria by inhibiting enoyl-ACP reductase of Plasmodium falciparum (vol 7,pg 167, 2000). Nat. Med. 2001, 7, 636. [CrossRef]

75. Waller, R.F.; Keeling, P.J.; Donald, R.G.K.; Striepen, B.; Handman, E.; Lang-Unnasch, N.; Cowman, A.F.; Besra, G.S.; Roos, D.S.; McFadden, G.I. Nuclear-encoded proteins target to the plastid in Toxoplasma gondii and Plasmodium falciparum. Proc. Natl. Acad. Sci. USA 1998, 95, 12352-12357. [CrossRef]

76. Botte, C.Y.; Yamaryo-Botte, Y.; Rupasinghe, T.W.T.; Mullin, K.A.; MacRae, J.I.; Spurck, T.P.; Kalanon, M.; Shears, M.J.; Coppel, R.L.; Crellin, P.K.; et al. Atypical lipid composition in the purified relict plastid (apicoplast) of malaria parasites. Proc. Natl. Acad. Sci. USA 2013, 110, 7506-7511. [CrossRef] [PubMed]

77. Goodman, C.D.; McFadden, G.I. Fatty acid synthesis in protozoan parasites: Unusual pathways and novel drug targets. Curr. Pharm. Des. 2008, 14, 901-916. [CrossRef] [PubMed]

78. Liu, B.Q.; Wang, Y.Q.; Fillgrove, K.L.; Anderson, V.E. Triclosan inhibits enoyl-reductase of type I fatty acid synthase in vitro and is cytotoxic to MCF-7 and SKBr-3 breast cancer cells. Cancer Chemother. Pharmacol. 2002, 49, 187-193. [CrossRef] [PubMed]

79. Kruger, N.J.; von Schaewen, A. The oxidative pentose phosphate pathway: Structure and organisation. Curr. Opin. Plant Biol. 2003, 6, 236-246. [CrossRef]

80. Bigogno, C.; Khozin-Goldberg, I.; Cohen, Z. Accumulation of arachidonic acid-rich triacylglycerols in the microalga Parietochloris incisa (Trebuxiophyceae, Chlorophyta). Phytochemistry 2002, 60, 135-143. [CrossRef]

81. Guschina, I.A.; Harwood, J.L. Mechanisms of temperature adaptation in poikilotherms. FEBS Lett. 2006, 580, 5477-5483. [CrossRef]

82. Khozin-Goldberg, I.; Didi-Cohen, S.; Shayakhmetova, I.; Cohen, Z. Biosynthesis of eicosapentaenoic acid (EPA) in the freshwater eustigmatophyte Monodus subterraneus (Eustigmatophyceae). J. Phycol. 2002, 38, 745-756. [CrossRef]

83. Mitschler, R.R.; Welti, R.; Upton, S.J. A comparative-study of lipid compositions of Cryptosporidium parvum (Apicomplexa) and madin-darby bovine kidney-cells. J. Eukaryot. Microbiol. 1994, 41, 8-12. [CrossRef]

84. Toso, M.A.; Omoto, C.K. Gregarina niphandrodes may lack both a plastid genome and organelle. J. Eukaryot. Microbiol. 2007, 54, 66-72. [CrossRef] [PubMed]

85. Zhu, G.; Marchewka, M.J.; Keithly, J.S. Cryptosporidium parvum appears to lack a plastid genome. Microbiology 2000, 146, 315-321. [CrossRef] [PubMed]

86. Keithly, J.S.; Langreth, S.G.; Buttle, K.F.; Mannella, C.A. Electron tomographic and ultrastructural analysis of the Cryptosporidium parvum relict mitochondrion, its associated membranes, and Organelles. J. Eukaryot. Microbiol. 2005, 52, 132-140. [CrossRef] [PubMed]

87. Ševčíková, T.; Horák, A.; Klimeš, V.; Zbránková, V.; Demir-Hilton, E.; Sudek, S.; Jenkins, J.; Schmutz, J.; Přibyl, P.; Fousek, J.; et al. Updating algal evolutionary relationships through plastid genome sequencing: Did alveolate plastids emerge through endosymbiosis of an ochrophyte? Sci. Rep. 2015, 5. [CrossRef] [PubMed]

88. Sobotka, R.; Esson, H.J.; Koník, P.; Trsková, E.; Moravcová, L.; Horák, A.; Dufková, P.; Oborník, M. Extensive gain and loss of photosystem I subunits in chromerid algae, photosynthetic relatives of apicomplexans. Sci. Rep. 2017, 7. [CrossRef] [PubMed]

(C) 2020 by the authors. Licensee MDPI, Basel, Switzerland. This article is an open access article distributed under the terms and conditions of the Creative Commons Attribution (CC BY) license (http://creativecommons.org/licenses/by/4.0/). 